\title{
THE OPTIMAL EXPONENT IN THE EMBEDDING INTO THE LEBESGUE SPACES FOR FUNCTIONS WITH GRADIENT IN THE MORREY SPACE
}

\author{
XAVIER CABRÉ AND FERNANDO CHARRO
}

\begin{abstract}
We study the following natural question that, apparently, has not been well addressed in the literature: Given functions $u$ with support in the unit ball $B_{1} \subset \mathbb{R}^{n}$ and with gradient in the Morrey space $M^{p, \lambda}\left(B_{1}\right)$, where $1<p<\lambda<n$, what is the largest range of exponents $q$ for which necessarily $u \in L^{q}\left(B_{1}\right)$ ? While David R. Adams proved in 1975 that this embedding holds for $q \leq \lambda p /(\lambda-p)$, an article from 2011 claimed the embedding in the larger range $q<n p /(\lambda-p)$. Here we disprove this last statement by constructing a function that provides a counterexample for $q>\lambda p /(\lambda-p)$. The function is basically a negative power of the distance to a set of Hausdorff dimension $n-\lambda$. When $\lambda \notin \mathbb{Z}$, this set is a fractal. We also make a detailed study of the radially symmetric case, a situation in which the exponent $q$ can go up to $n p /(\lambda-p)$.
\end{abstract}

\section{INTRODUCTION}

This article originated from the following natural question: Given functions $u$ with support in the unit ball $B_{1}(0) \subset \mathbb{R}^{n}$ and with gradient in the Morrey space $M^{p, \lambda}\left(B_{1}(0)\right.$ ), where $1<p<\lambda<n$, what is the optimal range of exponents $q$ such that necessarily $u \in L^{q}\left(B_{1}(0)\right)$ ? Apparently, this question has not been well addressed in the literature. In fact, the authors of [3, Theorem 2.5] claimed a range of exponents which, as we will prove in the current paper, turns out to be larger than the true one.

Our motivation came from the recent work [7] of the first author in collaboration with A. Figalli, X. Ros-Oton, and J. Serra, on the regularity of stable solutions to semilinear elliptic equations. Actually, the results of [7] are deduced from a Morrey type bound for the gradient of a stable solution, among other tools (see Remark 1.2 below for more details).

The following is the precise statement of the question that we are concerned with. Given real numbers $p$ and $\lambda$ such that

$$
1<p<\lambda<n,
$$

Key words and phrases. Morrey spaces, Optimal embeddings, Cantor sets.

2010 Mathematics Subject Classification: 42B37, 46E35.

The authors were supported by MINECO grant MTM2014-52402-C3-1-P. X. Cabré is also supported by MINECO grants MTM2017-84214-C2-1-P and MDM-2014-0445, and is member of the Catalan research group 2017 SGR 1392. F. Charro was also partially supported by a Juan de la Cierva fellowship. 
we wish to know for which exponents $q$ the inequality

$$
\|u\|_{L^{q}\left(B_{1}(0)\right)} \leq C\|\nabla u\|_{M^{p, \lambda}\left(B_{1}(0)\right)}
$$

holds true for functions $u$ with support in $B_{1}(0) \subset \mathbb{R}^{n}$ and for a constant $C$ independent of $u$, where

$$
\|\nabla u\|_{M^{p, \lambda}(\Omega)}^{p}:=\sup _{r>0, y \in \bar{\Omega}}\left(r^{\lambda-n} \int_{\Omega \cap B_{r}(y)}|\nabla u(x)|^{p} d x\right)
$$

is the Morrey norm of $\nabla u$ in a domain $\Omega \subset \mathbb{R}^{n}$. Notice that when $\lambda$ equals the dimension $n$ and $\Omega=B_{1}(0)$, (1.1) corresponds to the Sobolev inequality in $B_{1}(0) \subset \mathbb{R}^{n}$.

In 1975, D. R. Adams [1, Theorem 3.1] proved the following result. Let us denote in the sequel

$$
p_{1}:=\frac{\lambda p}{\lambda-p} \quad \text { and } \quad p_{2}:=\frac{n p}{\lambda-p} .
$$

Observe that, clearly, $p_{1}<p_{2}$.

Theorem 1.1 (D. R. Adams [1]). Let $p, \lambda \in \mathbb{R}$ satisfy $1<p<\lambda<n$ and let $u: \mathbb{R}^{n} \rightarrow \mathbb{R}$ be a Lipschitz function with $u \equiv 0$ in $\mathbb{R}^{n} \backslash B_{1}(0)$. Then, for every $q \leq p_{1}$, inequality (1.1) holds for a constant $C$ depending only on $n, p$, and $\lambda$.

For the reader's convenience, in Section 4 we will include his proof in this case $p>1$. The case $p=1$, which involves weak spaces, is also treated in [1].

In fact, Adams [1, Proposition 3.1 and Theorems 3.1 and 3.2] proved the following stronger embedding:

$$
\|u\|_{M^{p_{1}, \lambda}\left(B_{1}(0)\right)} \leq C\|\nabla u\|_{M^{p, \lambda}\left(B_{1}(0)\right)} .
$$

While inequality (1.2) is dimensionless by scaling, note that the dimensionless exponent for inequality (1.1) is $q=p_{2}$. This suggests that (1.1) could hold with $q=p_{2}$, or at least for $q<p_{2}$. In fact, it is easy to prove that among radially symmetric functions, (1.1) holds for every $q<p_{2}$ (see [5, Proposition 1.2(i)] and also Theorem 1.5] below).

In 2011, the authors of [3, Theorem 2.5] claimed that (1.1) held for every $q<p_{2}$ and for general functions, not necessarily radial. Some years later, we realized that the proof of [3, Theorem 2.5] was not correct. After that, the claim was withdrawn by the same authors in the Errata papers [4] and [5]. At the same time, we could not find other works addressing the exact question of what is the optimal exponent.

In the present paper we show that actually $q=p_{1}$ is the largest possible exponent in (1.1). To show this, for every $q>p_{1}$ we construct a non-radial function, described in detail below, for which $\|u\|_{L^{q}\left(B_{1}(0)\right)}=\infty$ while $\|\nabla u\|_{M^{p, \lambda}\left(B_{1}(0)\right)}<\infty$. An important feature of the function is that it depends only (up to a cutoff function) on $k$ variables $\left(x_{1}, \ldots, x_{k}\right)$, where $k$ is the smallest integer such that $\lambda \leq k$. The function is basically a negative power of the distance to a set of Hausdorff dimension $n-\lambda$. When $\lambda$ is not an integer this set is a fractal and, therefore, the structure of the function is not so "simple". In fact, it will be rather delicate to control the Morrey norm of its gradient. We could not find a simpler counterexample for $\lambda \notin \mathbb{Z}$, although we had several candidates that finally did not work. The possibility of finding simpler examples remains as an open question. 
In addition, we also consider a related norm, which we call the "triple norm", given by

$$
\left\|\left.\nabla \nabla\left|\|_{p, \lambda ; \Omega}^{p}:=\sup _{y \in \bar{\Omega}} \int_{\Omega}\right| \nabla u(x)\right|^{p}|x-y|^{\lambda-n} d x,\right.
$$

where $\Omega \subset \mathbb{R}^{n}$ is a domain. The article [7] on stable solutions to semilinear equations gives rise naturally to such a norm (see Remark 1.2 below) 1 Note that, clearly, we have

$$
\|\nabla u\|_{M^{p, \lambda}(\Omega)} \leq\|\nabla u\|_{p, \lambda ; \Omega}
$$

for every function $u$. The function that we construct will also satisfy $\|\nabla u\|_{p, \lambda ; B_{1}(0)}<\infty$, and thus $q=p_{1}$ is the largest possible exponent also for the embedding

$$
\|u\|_{L^{q}\left(B_{1}(0)\right)} \leq C\|\nabla u\|_{p, \lambda ; B_{1}(0)} .
$$

Instead, among radial functions we show that inequality (1.5) holds for every $q \leq p_{2}$, in contrast to inequality (1.1) for radial functions, which holds only for $q<p_{2}$.

Remark 1.2. The regularity results from the recent paper [7] on stable solutions to semilinear equations $-\Delta u=f(u)$ in a domain $\Omega \subset \mathbb{R}^{n}$ are based on bounds for a Morrey norm with $p=2$, of $\nabla u$ or, given a point $y$, of the radial derivative $\nabla u(x)$. $(x-y) /|x-y|$. For this, see [7, Lemma 2.1, step 2 in the proof of Theorem 1.2, and proof of Theorem 7.1], which also lead to the triple norm (1.3). The boundedness results from [7] up to dimension $n \leq 9$ correspond to $p=2$ and $\lambda=2$, while in the $L^{q}$ results for $n \geq 11$ one has $p=2<\lambda<n$ as in our paper. The results of the current article are used in [7] to determine optimally a range of exponents $q$ for which stable solutions necessarily belong to $L^{q}$ in dimensions $n \geq 11$.

Summarizing, our main contribution is the following result. It provides a counterexample to the validity of (1.1) and (1.5) for $q>p_{1}$, given by a function $u$ which is basically a negative power of the distance to a set of Hausdorff dimension $n-\lambda$. When $\lambda \notin \mathbb{Z}$, this set is a fractal.

Theorem 1.3. Let $p, \lambda \in \mathbb{R}$ satisfy $1<p<\lambda<n$. Then, for every $q>p_{1}:=$ $\lambda p /(\lambda-p)$ there exists a function $u: \mathbb{R}^{n} \rightarrow \mathbb{R}$ with $u \equiv 0$ in $\mathbb{R}^{n} \backslash B_{1}(0)$,

$$
\|u\|_{L^{q}\left(B_{1}(0)\right)}=\infty, \quad \text { and } \quad\|\nabla u\|_{p, \lambda ; B_{1}(0)}<\infty .
$$

In particular, we also have

$$
\|\nabla u\|_{M^{p, \lambda}\left(B_{1}(0)\right)}<\infty .
$$

If $\lambda$ is an integer, such function $u$ can be taken to be

$$
u(x)=\left(\left|x^{\prime}\right|^{-\alpha}-2^{\alpha}\right)+\xi\left(\left|x^{\prime \prime}\right|\right)
$$

where $x=\left(x^{\prime}, x^{\prime \prime}\right) \in \mathbb{R}^{\lambda} \times \mathbb{R}^{n-\lambda}$, the parameter $\alpha$ satisfies

$$
\frac{\lambda}{q} \leq \alpha<\frac{\lambda-p}{p}
$$

\footnotetext{
${ }^{1}$ The triple norm has been previously considered in the setting of the hole-filling technique for integral estimates; see [6. Section 1.2.3] among others. It also appears in [11, where it is called the Cordes-Nirenberg norm.
} 
and $\xi: \mathbb{R}^{+} \rightarrow[0,1]$ is a cutoff function with $\xi \equiv 1$ in $[0,1 / 2)$ and $\xi \equiv 0$ in $\mathbb{R}^{+} \backslash[0, \sqrt{3} / 3)$.

If $k-1<\lambda<k$ for some integer $k \in[2, n]$, the function $u$ can be taken to be

$$
u(x)= \begin{cases}\left(\operatorname{dist}\left(x, \mathcal{C}_{n, \lambda}\right)^{-\alpha}-4^{\alpha}\right)_{+} & \text {if } k=n \\ \left(\operatorname{dist}\left(x^{\prime}, \mathcal{C}_{k, \lambda}\right)^{-\alpha}-4^{\alpha}\right)_{+} \xi\left(\left|x^{\prime \prime}\right|\right) & \text { if } k<n,\end{cases}
$$

where $x=\left(x^{\prime}, x^{\prime \prime}\right) \in \mathbb{R}^{k} \times \mathbb{R}^{n-k}$, $\alpha$ satisfies (1.8), $\xi$ is a cutoff function as above, and $\mathcal{C}_{k, \lambda}$ is a set of Hausdorff dimension $k-\lambda$ in $\mathbb{R}^{k}$ given by

$$
\mathcal{C}_{k, \lambda}=\{0\} \times C_{\gamma} \subset \mathbb{R}^{k-1} \times[-1 / 2,1 / 2],
$$

where $C_{\gamma} \subset[-1 / 2,1 / 2]$ is the generalized Cantor set with parameter $\gamma=1-2^{1-\frac{1}{k-\lambda}}$ defined in the following remark.

We emphasize that the counterexample to the embedding is therefore given by a function that, up to a cutoff, only depends on $k$ variables, where $k$ is the smallest integer such that $\lambda \leq k$.

Remark 1.4. The generalized Cantor set $C_{\gamma}$ (see [9]) is obtained from the interval $[-1 / 2,1 / 2]$ by removing at iteration $j=1,2, \ldots$ the central interval of length $\gamma l_{j-1}$ from each remaining segment of length $l_{j-1}=((1-\gamma) / 2)^{j-1}$; see Figure 2 in Section 6 . The usual Cantor set corresponds to $\gamma=1 / 3$. The reason for our choice of $\gamma$ is that the Hausdorff dimension of $C_{\gamma}$ is

$$
\frac{-\log 2}{\log \frac{1-\gamma}{2}}=k-\lambda \in(0,1)
$$

(see [9, Theorem 9.3]). In particular, letting $\lambda$ range from $k-1$ to $k$ yields any fractal dimension between 0 and 1 , and (1.9) somehow interpolates between the integer cases $\lambda=k-1$ and $\lambda=k$.

Let us describe briefly how we found that $p_{1}$ is the optimal exponent. In the case when $\lambda$ is an integer, the hint came from the number $p_{1}=\lambda p /(\lambda-p)$, which can be thought of as the Sobolev exponent in dimension $\lambda$. It was natural then to choose the function (1.7), since it gives a counterexample for the Sobolev inequality in $\mathbb{R}^{\lambda}$ when $q>\lambda p /(\lambda-p)=p_{1}$ and the exponent $\alpha$ is chosen appropriately.

When $\lambda \in \mathbb{Z}$, (1.7) is basically a negative power of the distance to a subspace of dimension $n-\lambda$. Therefore, when $\lambda \notin \mathbb{Z}$, a negative power of the distance to a set of Hausdorff dimension $n-\lambda$ became a natural candidate to counterexample. This is what the function in (1.9) basically is, a power of the distance to $\mathcal{C}_{k, \lambda} \times \mathbb{R}^{n-k}$.

It may be of interest to recall here the solutions found by R. Schoen and S.-T. Yau in [12, Section 5] for nonlinear equations with critical exponent. They construct weak solutions which are singular on a Cantor set with fractional Hausdorff dimension; see [12, Page 70]. Obviously, nonlinear equations with critical exponent are closely related to the Sobolev embedding. Another result on solutions with a singular set of Cantor type is due to Fonseca, Malý, and Mingione [10], a paper that concerns the minimizers of a certain scalar, convex, and regular Lagrangian.

The paper 2 2 by Adams and Lewis was brought to our attention after the completion of the current article. In [2], the authors proved that functions which satisfy an 
integrability condition of Morrey-Besov type belong also to a certain Lorentz space. In addition, they construct examples of functions to show that their embeddings are the best possible. The Morrey-Besov norm is a fractional Morrey-type condition involving the $\alpha$-th difference quotients of a function, where $0<\alpha<1$. Thus, this concerns more exotic norms than the basic and standard ones that we treat. The authors of [2] mention the possibility that the ideas in their proof of [2, Theorem 3] could be extended from the case $\alpha \in(0,1)$ that they treat to the case $\alpha=1$ (and $p=q$ in their paper). This is something that we have not explored. There could be the usual delicate issues taking limits of fractional integral norms as $\alpha \rightarrow 1$, or even simply the impossibility of taking this limit or adapting the proof for $\alpha=1$. However, if this could be done, it would show that actually $q=p_{1}$ is the largest possible exponent in (1.1). On the other hand, it is not clear at all if their example would allow to recover our result on the optimal exponent for the embedding (1.5) concerning the "triple" norm.

Among radial functions, the optimal ranges of exponents in inequalities (1.1) and (1.5) are strictly larger than those of Theorems 1.1 and 1.3. This is the content of the following result, where we show that the exponent $q$ can go up to $q_{2}$. Interestingly, here the answer is different for the Morrey and the "triple" norms: we prove that (1.1) is false for $q=p_{2}$ while (1.5) holds for this exponent. Here we can include the exponent $p=1$.

Theorem 1.5. Let $p, \lambda \in \mathbb{R}$ satisfy $1 \leq p<\lambda<n$, and let $p_{2}:=n p /(\lambda-p)$.

(a) For every $1 \leq q<p_{2}$ and all radially symmetric $C^{1}$ functions $u$ vanishing on $\partial B_{1}(0)$, we have

$$
\|u\|_{L^{q}\left(B_{1}(0)\right)} \leq C\|\nabla u\|_{M^{p, \lambda}\left(B_{1}(0)\right)},
$$

where $C$ is a constant depending only on $n, p, \lambda$, and $q$. In addition, this embedding is false for $q \geq p_{2}$.

(b) For all radially symmetric $C^{1}$ functions $u$ with compact support in $\mathbb{R}^{n}$, we have

$$
\|u\|_{L^{p_{2}\left(\mathbb{R}^{n}\right)}} \leq C\|\| \nabla u \|_{p, \lambda ; \mathbb{R}^{n}},
$$

where $C$ is a constant depending only on $n, p$, and $\lambda$. In addition, $p_{2}$ is the optimal exponent in this inequality.

The paper is organized as follows. In Section 2, we prove a monotonicity result, Lemma 2.1, that we will use several times throughout the paper to optimize the location of the "singularities" $y$ in the Morrey and "triple" norms, both in the radial and nonradial cases. In Section 3 we prove the embeddings for radial functions, Theorem 1.5 . In Section 4 we provide for the reader's convenience D. R. Adams' [1] proof of Theorem 1.1 in the general case of non-radial functions. In Sections 5 and 6 we prove Theorem 1.3 on the optimality of the embeddings. We consider separately the case when $\lambda$ is an integer in Section 5 (for its simplicity) and the case when $\lambda$ is a non-integer in Section 6] (which is much more involved).

Notation. In the sequel $B_{R}^{(m)}(x)$ denotes the open ball in $\mathbb{R}^{m}$ of radius $R$ centered at $x$. For simplicity, whenever $m$ or $x$ are omitted, we will consider $m=n$ and $x=0$ respectively. By $C$, we denote constants that may change from line to line. For points in $\mathbb{R}^{n}$, we will write $x=\left(x^{\prime}, x^{\prime \prime}\right) \in \mathbb{R}^{k} \times \mathbb{R}^{n-k}$ for $k$ a positive integer specified from the 
context. Given a function $u, u_{+}=\max \{u, 0\}$ is its positive part. As mentioned before, we will denote $p_{1}=\lambda p /(\lambda-p)$ and $p_{2}=n p /(\lambda-p)$. For convenience, we will use the following standard notation for intervals: $a(-b, b)+h=(h-a b, h+a b)$. Finally, $\operatorname{dist}(t, U)=\inf _{z \in U}|t-z|$ as usual.

\section{ON THE LOCATION OF THE SINGULARITY IN THE "TRIPLE" NORM}

In this section we prove a monotonicity result that we will use several times in the sequel to study which locations of the "singularity" $y$ make larger the integral in the "triple norm"

$$
\|\nabla u\|_{p, \lambda ; \Omega}^{p}=\sup _{y \in \bar{\Omega}} \int_{\Omega}|\nabla u(x)|^{p}|x-y|^{\lambda-n} d x .
$$

Lemma 2.1. Consider a domain $\Omega \subset \mathbb{R}^{n}$, convex in the $e_{1}$ direction, and symmetric with respect to $\left\{z_{1}=0\right\}$. Let $J: \mathbb{R}^{n} \rightarrow \mathbb{R}$ be given by

$$
J(y):=\int_{\Omega} h(z)|z-y|^{-\theta} d z
$$

with $\theta>0$ and $h$ a non-negative function in $\Omega$. Then:

(a) $J$ is non-increasing with respect to $y_{1}$ in $\left\{y_{1} \geq \sup _{z \in \Omega} z_{1}\right\}$, and non-decreasing with respect to $y_{1}$ in $\left\{y_{1} \leq \inf _{z \in \Omega} z_{1}\right\}$.

(b1) Suppose that the non-negative function $h$ satisfies that, for some $\eta \in\left[0, \sup _{z \in \Omega} z_{1}\right)$ and every $y_{1} \in\left[\eta, \sup _{z \in \Omega} z_{1}\right)$,

$$
h\left(z^{*}\right) \geq h(z) \quad \text { for all } z \in \Omega \cap\left\{z_{1} \geq y_{1}\right\}
$$

where $z^{*}=\left(2 y_{1}-z_{1}, z^{\prime}\right) \in \mathbb{R} \times \mathbb{R}^{n-1}$ is the reflection of $z$ with respect to the hyperplane $\left\{z_{1}=y_{1}\right\}$. Then, $J$ is non-increasing with respect to $y_{1}$ in $\left\{\eta \leq y_{1}<\sup _{z \in \Omega} z_{1}\right\}$.

(b2) On the other hand, if the non-negative function $h$ is such that, for some $\eta \in$ $\left(\inf _{z \in \Omega} z_{1}, 0\right]$ and every $y_{1} \in\left(\inf _{z \in \Omega} z_{1}, \eta\right]$,

$$
h\left(z^{*}\right) \geq h(z) \quad \text { for all } z \in \Omega \cap\left\{z_{1} \leq y_{1}\right\}
$$

with $z^{*}$ as before, then $J$ is non-decreasing with respect to $y_{1}$ in $\left\{\inf _{z \in \Omega} z_{1}<\right.$ $\left.y_{1} \leq \eta\right\}$.

Proof. Observe first that for every $z \in \Omega$, the quantity $|z-y|$ is increasing with respect to $y_{1}$ in $\left\{y_{1} \geq \sup _{z \in \Omega} z_{1}\right\}$, and decreasing with respect to $y_{1}$ in $\left\{y_{1} \leq \inf _{z \in \Omega} z_{1}\right\}$. Since $h \geq 0$ and $\theta>0$, we deduce that $J$ is non-increasing with respect to $y_{1}$ in $\left\{y_{1} \geq \sup _{z \in \Omega} z_{1}\right\}$, and non-decreasing with respect to $y_{1}$ in $\left\{y_{1} \leq \inf _{z \in \Omega} z_{1}\right\}$. This proves part (a). 


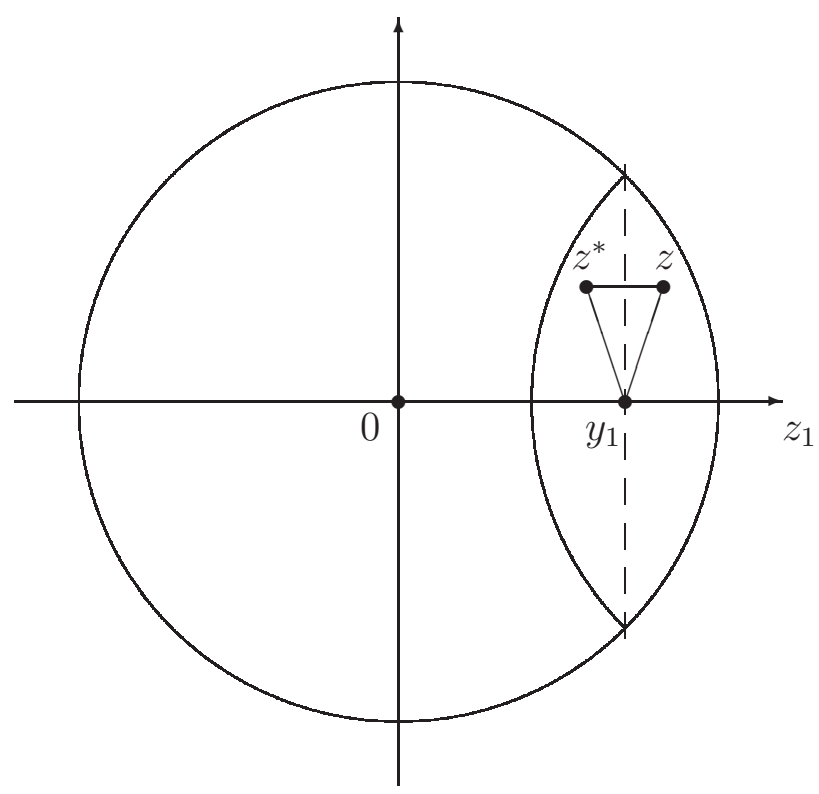

Figure 1. Monotonicity argument in the proof of Lemma 2.1.

Assume now that $0 \leq \eta \leq y_{1}<\sup _{z \in \Omega} z_{1}$ and compute

$$
\begin{aligned}
\partial_{y_{1}} J(y)=\theta & \int_{\Omega} h(z)\left(z_{1}-y_{1}\right)|z-y|^{-\theta-2} d z \\
=\theta & \left\{\int_{\Omega \cap\left\{z_{1} \geq y_{1}\right\}} h(z)\left(z_{1}-y_{1}\right)|z-y|^{-\theta-2} d z\right. \\
& \left.+\int_{\Omega \cap\left\{z_{1} \leq y_{1}\right\}} h(z)\left(z_{1}-y_{1}\right)|z-y|^{-\theta-2} d z\right\} .
\end{aligned}
$$

For every $z \in \Omega \cap\left\{z_{1} \geq y_{1}\right\}$, let $z^{*}=\left(2 y_{1}-z_{1}, z^{\prime}\right)$ be its reflection with respect to the hyperplane $\left\{z_{1}=y_{1}\right\}$; see Figure 1. Then, $\left|z^{*}-y\right|=|z-y|$, while $h\left(z^{*}\right) \geq h(z)$ by hypothesis. Therefore, for every $z \in \Omega \cap\left\{z_{1} \geq y_{1}\right\}$, we have

$$
h(z)\left(z_{1}-y_{1}\right)|z-y|^{-\theta-2} \leq-h\left(z^{*}\right)\left(z_{1}^{*}-y_{1}\right)\left|z^{*}-y\right|^{-\theta-2}
$$

and hence, using that $h \geq 0$,

$$
\begin{aligned}
\int_{\Omega \cap\left\{z_{1} \geq y_{1}\right\}} & h(z)\left(z_{1}-y_{1}\right)|z-y|^{-\theta-2} d z \\
& \leq-\int_{\left(\Omega \cap\left\{z_{1} \geq y_{1}\right\}\right)^{*}} h(z)\left(z_{1}-y_{1}\right)|z-y|^{-\theta-2} d z \\
& \leq-\int_{\Omega \cap\left\{z_{1} \leq y_{1}\right\}} h(z)\left(z_{1}-y_{1}\right)|z-y|^{-\theta-2} d z .
\end{aligned}
$$


Therefore, $\partial_{y_{1}} J(y) \leq 0$ for all $\eta \leq y_{1}<\sup _{z \in \Omega} z_{1}$ and the conclusion in part (b1) follows. The statement for $\inf _{z \in \Omega} z_{1}<y_{1} \leq \eta$ in part (b2) follows from (b1) by reflection.

\section{The radial case: Proof of Theorem 1.5}

In this section we establish Theorem 1.5 on the embeddings for radial functions. In the proof we apply Lemma 2.1 (the monotonicity result proved in Section 2), which will also be used for the non-radial case in Section 6. We point out that the Sobolev inequalities with monomial weights established in [8] by Ros-Oton and the first author will be of great use.

Proof of Theorem [1.5. We structure the proof in four parts. In Part 1 we establish estimate (1.10), while in Part 2 we show that $q<p_{2}$ is the optimal range of exponents for this estimate. In Part 3a we prove (1.11); here we will use the results of [8]. Part $3 \mathrm{~b}$ provides an alternative proof of (1.11). Finally, we show in Part 4 that $p_{2}$ is the largest exponent for which (1.11) holds.

Part 1. We proceed now to show estimate (1.10) for $1 \leq q<p_{2}$. All the constants $C$ will depend only on $n, p, \lambda$, and $q$. On the one hand we have

$$
\begin{aligned}
& \|u\|_{L^{q}\left(B_{1}(0)\right)}^{q}=C \int_{0}^{1}|u(r)|^{q} r^{n-1} d r \\
& \leq C \int_{0}^{1}\left(\int_{r}^{1}\left|u^{\prime}(s)\right| d s\right)^{q} r^{n-1} d r=C \sum_{j=1}^{\infty} \int_{2^{-j}}^{2^{1-j}}\left(\int_{r}^{1}\left|u^{\prime}(s)\right| d s\right)^{q} r^{n-1} d r \\
& \leq C \sum_{j=1}^{\infty} \int_{2^{-j}}^{2^{1-j}}\left(\int_{2^{-j}}^{1}\left|u^{\prime}(s)\right| d s\right)^{q} r^{n-1} d r=C \frac{2^{n}-1}{n} \sum_{j=1}^{\infty} 2^{-j n}\left(\int_{2^{-j}}^{1}\left|u^{\prime}(s)\right| d s\right)^{q} .
\end{aligned}
$$

Now, Hölder's inequality yields

$$
\begin{aligned}
\int_{2^{-j}}^{1}\left|u^{\prime}(s)\right| d s & =\sum_{i=1}^{j} \int_{2^{-i}}^{2^{1-i}}\left|u^{\prime}(s)\right| d s \leq \sum_{i=1}^{j} 2^{-i \frac{p-1}{p}}\left(\int_{2^{-i}}^{2^{1-i}}\left|u^{\prime}(s)\right|^{p} d s\right)^{\frac{1}{p}} \\
& \leq \sum_{i=1}^{j} 2^{-i \frac{p-1}{p}+i \frac{n-1}{p}}\left(\int_{2^{-i}}^{2^{1-i}}\left|u^{\prime}(s)\right|^{p} s^{n-1} d s\right)^{\frac{1}{p}} \\
& \left.\leq C \sum_{i=1}^{j} 2^{-i \frac{p-n}{p}}\left(\int_{B_{2^{1-i}}(0)}|\nabla u(x)|^{p} d x\right)^{\frac{1}{p}}|\nabla u(x)|^{p} d x\right)^{\frac{1}{p}} \\
& =C \sum_{i=1}^{j} 2^{-i \frac{p-n}{p}+(1-i) \frac{n-\lambda}{p}}\left(2^{(1-i)(\lambda-n)} \int_{B_{2^{1-i}}(0) \cap B_{1}(0)} \mid \Delta \sum_{i=1}^{j} 2^{i \frac{\lambda-p}{p}}\|\nabla u\|_{M^{p, \lambda}\left(B_{1}(0)\right)} \leq C 2^{j \frac{\lambda-p}{p}}\|\nabla u\|_{M^{p, \lambda}\left(B_{1}(0)\right)} .\right.
\end{aligned}
$$


Therefore, we obtain

$$
\|u\|_{L^{q}\left(B_{1}(0)\right)}^{q} \leq C \sum_{j=1}^{\infty} 2^{j \frac{q(\lambda-p)-n p}{p}}\|\nabla u\|_{M^{p, \lambda}\left(B_{1}(0)\right)}^{q},
$$

and the series is convergent since $q<p_{2}$.

Part 2. In order to show that $q<p_{2}$ is the optimal range of exponents in estimate (1.10), let $q \geq p_{2}$ and consider the function $u_{\alpha}(x)=|x|^{-\alpha}-1$, extended by zero outside $B_{1}(0)$, with $\alpha=(\lambda-p) / p$. Notice that $u_{\alpha}$ vanishes on $\partial B_{1}(0)$ and that $\left\|u_{\alpha}\right\|_{L^{q}\left(B_{1}(0)\right)}=\infty$, since $n \leq \alpha q$.

To show that $\left\|\nabla u_{\alpha}\right\|_{M^{p, \lambda}\left(B_{1}(0)\right)}$ is finite, let $y \in \bar{B}_{1}(0)$ and $r>0$. Observe that we can write $-y$ instead of $y$ in the definition of the Morrey norm. Then,

$$
\begin{aligned}
\int_{B_{1}(0) \cap B_{r}(-y)}\left|\nabla u_{\alpha}(x)\right|^{p} d x & =C \int_{B_{1}(0) \cap B_{r}(-y)}|x|^{-\alpha p-p} d x \\
& \leq C \int_{B_{r}(-y)}|x|^{-\alpha p-p} d x=C \int_{B_{r}(0)}|z-y|^{-\alpha p-p} d z
\end{aligned}
$$

by the change of variables $z=x+y$. Notice that, upon a rotation, we can assume $y=y_{1} e_{1}=\left(y_{1}, 0, \ldots, 0\right)$ with $y_{1} \geq 0$. Denote

$$
J\left(y_{1}\right)=\int_{B_{r}(0)}\left|z-y_{1} e_{1}\right|^{-\alpha p-p} d z .
$$

We can now apply Lemma 2.1 (with $\eta=0$ and $h \equiv 1$ ) and conclude that $J$ is nonincreasing in $[0, \infty)$. Therefore, $J\left(y_{1}\right) \leq J(0)$ for all $y_{1} \geq 0$.

As a consequence, we have that

$$
r^{\lambda-n} \int_{B_{1}(0) \cap B_{r}(-y)}\left|\nabla u_{\alpha}(x)\right|^{p} d x \leq C r^{\lambda-n} \int_{0}^{r} s^{n-\alpha p-p-1} d s \leq C
$$

independently of $r$, by our choice of $\alpha$.

Part 3a. We give here a first proof of estimate (1.11). Recall that $u$ has compact support. Since $u$ is radial, it suffices to show that

$$
\left(\int_{0}^{\infty}|u(r)|^{\frac{n p}{\lambda-p}} r^{n-1} d r\right)^{\frac{\lambda-p}{n p}} \leq C\left(\int_{0}^{\infty}\left|u^{\prime}(r)\right|^{p} r^{\lambda-1} d r\right)^{\frac{1}{p}} .
$$

In fact, we are going to prove (3.1) with the best constant. For this, we perform the change of variables $r=s^{a}$ with $a=p /(n-\lambda+p)$ and $v(s)=u\left(s^{a}\right)$ in the integrals on both sides of (3.1). We get

$$
\int_{0}^{\infty}|u(r)|^{\frac{n p}{\lambda-p}} r^{n-1} d r=a \int_{0}^{\infty}|v(s)|^{\frac{n p}{\lambda-p}} s^{a n-1} d s
$$

and

$$
\int_{0}^{\infty}\left|u^{\prime}(r)\right|^{p} r^{\lambda-1} d r=a^{1-p} \int_{0}^{\infty}\left|v^{\prime}(s)\right|^{p} s^{a n-1} d s .
$$

Observe that after the change of variables, both integrals are weighted by the same power an $-1 \geq 0$. In this way, (3.1) becomes a Bliss type inequality that was proved by 
Talenti [13]. From [13, Lemma 2] (or also from [8, Theorem 1.3] applied in dimension 1 with $D=A_{1}+1=$ an) we obtain that

$$
\left(\int_{0}^{\infty}|v(s)|^{\frac{n p}{\lambda-p}} s^{a n-1} d s\right)^{\frac{\lambda-p}{n p}} \leq C_{p}\left(\int_{0}^{\infty}\left|v^{\prime}(s)\right|^{p} s^{a n-1} d s\right)^{\frac{1}{p}},
$$

with an explicit value of its best constant $C_{p}$. Inequality (3.1), and thus (1.11), is now established.

Moreover, it is also shown in [13, Lemma 2] (see also [8]) that when $1<p<\lambda$ the constant $C_{p}$ is attained in $W_{0}^{1, p}\left(\mathbb{R},|s|^{a n-1} d s\right)$, the closure of $C_{c}^{1}(\mathbb{R})$ under the norm $\left(\int_{\mathbb{R}}\left(|u|^{p}+|\nabla u|^{p}\right)|s|^{a n-1} d s\right)^{1 / p}$, by the functions

$$
v(s)=\left(c_{1}+c_{2}|s|^{\frac{p}{p-1}}\right)^{\frac{p-\lambda}{n+p-\lambda}}
$$

where $c_{1}, c_{2}>0$ are arbitrary constants. On the other hand, when $p=1$, the constant $C_{1}$ is not attained by any function in $W_{0}^{1,1}\left(\mathbb{R},|s|^{a n-1} d s\right)$. Note however, that knowing the best constant for (3.1) does not ensure that we know the best constant for (1.11). Indeed, for

$$
u_{c_{1}, c_{2}}(x)=\left(c_{1}+c_{2}|x|^{\frac{n+p-\lambda}{p-1}}\right)^{\frac{p-\lambda}{n+p-\lambda}}, \quad c_{1}, c_{2}>0
$$

we have seen that

$$
\left\|u_{c_{1}, c_{2}}\right\|_{L^{p_{2}\left(\mathbb{R}^{n}\right)}}=C \int_{\mathbb{R}^{n}}\left|\nabla u_{c_{1}, c_{2}}(x)\right|^{p}|x|^{\lambda-n} d x
$$

with the best constant $C$, but on the other hand we do not know if the supremum in the "triple norm" is attained at $y=0$. That is, we do not know if

$$
\int_{\mathbb{R}^{n}}\left|\nabla u_{c_{1}, c_{2}}(x)\right|^{p}|x|^{\lambda-n} d x=\left\||| \nabla u_{c_{1}, c_{2}} \mid\right\|_{p, \lambda ; \mathbb{R}^{n}} .
$$

The point here is that $\left|\nabla u_{c_{1}, c_{2}}\right|^{p}$ is zero at the origin, instead of blowing up as in the other cases that we consider in the paper. Therefore,

$$
\int_{\mathbb{R}^{n}}\left|\nabla u_{c_{1}, c_{2}}(x)\right|^{p}|x-y|^{\lambda-n} d x
$$

could perhaps be increasing in $|y|$ in an interval near the origin, and then decrease to 0 as $|y| \rightarrow \infty$. In this setting, the reflection argument in the proof of Lemma 2.1 does not work and we cannot conclude (3.2) as before.

Part 3b. We will provide here an alternative proof of estimate (1.11). First, we establish the case $p=1$ by proving

$$
\left(\int_{0}^{\infty}|u(r)|^{\frac{n}{\lambda-1}} r^{n-1} d r\right)^{\frac{\lambda-1}{n}} \leq C \int_{0}^{\infty}\left|u^{\prime}(r)\right| r^{\lambda-1} d r
$$

for every $\lambda \in(1, n)$. One can then deduce the general case applying (3.3) with $(n(p-$ $1)+\lambda) / p$ instead of $\lambda$ to the function $u^{b}$ with $b=1+n(p-1) /(\lambda-p)$. Note that $1<(n(p-1)+\lambda) / p<n$. 
To show (3.3), notice that we can assume $u \geq 0$. Furthermore, we can also assume that $u$ is radially decreasing. This follows from [8, Proposition 4.2] (see also [14]) applied to inequality (3.3) after changing variables as in Part 3 in order to guarantee that both sides of the inequality are weighted by the same power. Now, on the one hand, the change of variables $t=u(r)$ yields

$$
\int_{0}^{\infty}\left|u^{\prime}(r)\right| r^{\lambda-1} d r=-\int_{0}^{\infty} u^{\prime}(r) r^{\lambda-1} d r=\int_{0}^{\max (u)} \varphi(t)^{\frac{\lambda-1}{n}} d t
$$

for $\varphi(t)=|\{r: u(r)>t\}|^{n}$. On the other hand, by Cavalieri's principle

$$
\int_{0}^{\infty} u(r)^{\frac{n}{\lambda-1}} r^{n-1} d r=\frac{1}{n} \int_{0}^{\infty} \frac{n}{\lambda-1} t^{\frac{n}{\lambda-1}-1} \varphi(t) d t .
$$

We conclude by proving that

$$
\int_{0}^{\infty} q t^{q-1} \varphi(t) d t \leq\left(\int_{0}^{\infty} \varphi(t)^{\frac{1}{q}} d t\right)^{q}
$$

for every non-increasing function $\varphi=\varphi(t)$ and every $q>1$. Consequently, (3.3) will follow from (3.4), (3.5), and (3.6). To prove inequality (3.6), denote

$$
F_{1}(s)=\int_{0}^{s} q t^{q-1} \varphi(t) d t \quad \text { and } \quad F_{2}(s)=\left(\int_{0}^{s} \varphi(t)^{\frac{1}{q}} d t\right)^{q},
$$

and notice that $F_{1}(0)=F_{2}(0)$, while

$$
\begin{aligned}
F_{2}^{\prime}(s) & =q \varphi(s)^{\frac{1}{q}}\left(\int_{0}^{s} \varphi(t)^{\frac{1}{q}} d t\right)^{q-1} \\
& \geq q \varphi(s)^{\frac{1}{q}}\left(s \varphi(s)^{\frac{1}{q}}\right)^{q-1}=q s^{q-1} \varphi(s)=F_{1}^{\prime}(s) .
\end{aligned}
$$

This establishes (3.6) and hence concludes the proof of (1.11).

Part 4. Finally, we show that $p_{2}$ is the largest exponent for which (1.11) holds. Let $q>p_{2}$ and consider the function $u_{\alpha}(x)=\left(|x|^{-\alpha}-1\right)_{+}$with $n / q \leq \alpha<(\lambda-p) / p$. Notice that

$$
\left\|u_{\alpha}\right\|_{L^{q}\left(\mathbb{R}^{n}\right)}^{q}=\int_{B_{1}(0)}\left(|x|^{-\alpha}-1\right)^{q} d x=C \int_{0}^{1}\left(1-r^{\alpha}\right)^{q} r^{n-\alpha q-1} d r=\infty,
$$

by our choice of $\alpha$.

On the other hand, we are going to see that $\left\|\nabla u_{\alpha}\right\| \|_{p, \lambda ; \mathbb{R}^{n}}<\infty$, thus contradicting the inequality. To prove this, we claim that $y=0$ realizes the supremum in the definition of $\left\|\mid \nabla u_{\alpha}\right\|_{p, \lambda ; \mathbb{R}^{n}}$, and hence

$$
\left\|\nabla u_{\alpha}\right\|_{p, \lambda ; \mathbb{R}^{n}}^{p}=\int_{B_{1}(0)}\left|\nabla u_{\alpha}(x)\right|^{p}|x|^{\lambda-n} d x=C \int_{0}^{1} r^{\lambda-\alpha p-p-1} d r<\infty,
$$

since $\lambda-\alpha p-p>0$.

We conclude proving the claim by monotonicity. For $y \in \mathbb{R}^{n}$, we have that

$$
\int_{B_{1}(0)}\left|\nabla u_{\alpha}(x)\right|^{p}|x-y|^{\lambda-n} d x=\alpha^{p} \int_{B_{1}(0)}|x|^{-\alpha p-p}|x-y|^{\lambda-n} d x .
$$


Notice that, upon a rotation, we can assume $y=y_{1} e_{1}=\left(y_{1}, 0, \ldots, 0\right)$. Denote

$$
J\left(y_{1}\right)=\int_{B_{1}(0)}|x|^{-\alpha p-p}\left|x-y_{1} e_{1}\right|^{\lambda-n} d x .
$$

Since the function $J$ is under the hypotheses of Lemma 2.1 (with $\eta=0$ ), we conclude that $J$ is non-increasing in $[0, \infty)$, and therefore that $J\left(y_{1}\right) \leq J(0)$ for all $y_{1} \geq 0$.

\section{Embeddings in the general Case: Proof of Theorem 1.1}

For the reader's convenience, we provide in this section D. R. Adams' proof of Theorem 1.1, see [1, Theorem 3.1]. Recall that we consider a Lipschitz function $u: \mathbb{R}^{n} \rightarrow \mathbb{R}$ with $u \equiv 0$ in $\mathbb{R}^{n} \backslash B_{1}(0)$, so that integrals in $\mathbb{R}^{n}$ and integrals in $B_{1}(0)$ coincide.

Proof of Theorem 1.1. The proof is based on the following two claims,

$$
|u(x)| \leq C\left(I_{1}|\nabla u|\right)(x), \quad \text { a.e. } x .
$$

and

where

$$
\left|I_{1}\right| \nabla u||(x) \leq C\left(M_{\lambda / p}|\nabla u|(x)\right)^{\frac{p}{\lambda}}\left(M_{0}|\nabla u|(x)\right)^{1-\frac{p}{\lambda}}
$$

$$
I_{1} f(x):=\int_{\mathbb{R}^{n}} f(y)|y-x|^{1-n} d y
$$

is the Riesz potential of $f$, and

$$
M_{\beta} f(x):=\sup _{r>0}\left(r^{\beta-n} \int_{B_{r}(x)}|f(z)| d z\right), \quad 0 \leq \beta \leq n
$$

is the maximal function with parameter $\beta$.

Once (4.1) and (4.2) are established, we can finish the proof as follows. By Hölder's inequality, we have

$$
\begin{aligned}
M_{\lambda / p}|\nabla u|(x) & =\sup _{r>0}\left(r^{\frac{\lambda}{p}-n} \int_{B_{r}(x)}|\nabla u(z)| d z\right) \\
& \leq C \sup _{r>0}\left(r^{\lambda-n} \int_{B_{1}(0) \cap B_{r}(x)}|\nabla u(z)|^{p} d z\right)^{\frac{1}{p}} \leq C\|\nabla u\|_{M^{p, \lambda}\left(B_{1}(0)\right)} .
\end{aligned}
$$

(recall that $u \equiv 0$ in $\mathbb{R}^{n} \backslash B_{1}(0)$ ). Then, (4.1) and (4.2) give

$$
|u(x)| \leq C\left(M_{\lambda / p}|\nabla u|(x)\right)^{\frac{p}{\lambda}}\left(M_{0}|\nabla u|(x)\right)^{1-\frac{p}{\lambda}} \leq C\|\nabla u\|_{M^{p, \lambda}\left(B_{1}(0)\right)}^{\frac{p}{\lambda}}\left(M_{0}|\nabla u|(x)\right)^{1-\frac{p}{\lambda}}
$$

almost everywhere. It follows that

$$
\begin{aligned}
\|u\|_{L^{q}\left(B_{1}(0)\right)}^{q} & \leq C^{q}\|\nabla u\|_{M^{p, \lambda}\left(B_{1}(0)\right)}^{\frac{p q}{\lambda}} \int_{B_{1}(0)}\left(M_{0}|\nabla u|(x)\right)^{\frac{p q}{p_{1}}} d x \\
& \leq C^{q}\|\nabla u\|_{M^{p, \lambda}\left(B_{1}(0)\right)}^{\frac{p q}{\lambda}}\left|B_{1}(0)\right|^{1-\frac{q}{p_{1}}}\left\|M_{0}|\nabla u|\right\|_{L^{p}\left(B_{1}(0)\right)}^{\frac{p q}{p_{1}}},
\end{aligned}
$$

where we have applied Hölder's inequality with exponents $p_{1} /\left(p_{1}-q\right)$ and $p_{1} / q$.

By the well-known $L^{p}$ estimate for the maximal function $M_{0}$ when $p>1$, there exists a constant $C$ depending only on $n$ and $p$ such that

$$
\left\|M_{0}|\nabla u|\right\|_{L^{p}\left(B_{1}(0)\right)} \leq C\|\nabla u\|_{L^{p}\left(B_{1}(0)\right)},
$$


and therefore

$$
\|u\|_{L^{q}\left(B_{1}(0)\right)} \leq C\left|B_{1}(0)\right|^{\frac{1}{q}-\frac{1}{p_{1}}}\|\nabla u\|_{M^{p, \lambda}\left(B_{1}(0)\right)}^{\frac{p}{\lambda}+\frac{p}{p_{1}}} \leq C\|\nabla u\|_{M^{p, \lambda}\left(B_{1}(0)\right)}
$$

with $C$ depending only on $n, p$, and $\lambda$ as desired.

Therefore, it remains to prove claims (4.1) and (4.2).

Consider first estimate (4.1). To prove it, notice that for $\sigma \in \mathbb{R}^{n}$ with $|\sigma|=1$

$$
|u(x)|=\left|-\int_{0}^{\infty} \frac{d}{d r} u(x+r \sigma) d r\right| \leq \int_{0}^{\infty}|\nabla u(x+r \sigma)| d r .
$$

Then, integrating on $\sigma$ we get

$$
|u(x)| \leq C \int_{0}^{\infty} \int_{\partial B_{1}(0)}\left(|\nabla u(x+r \sigma)| r^{1-n}\right) r^{n-1} d \sigma d r=C\left(I_{1}|\nabla u|\right)(x)
$$

and (4.1) is proved.

Next, consider estimate (4.2). We reproduce the argument in [1] to show that for a given function $f$ with compact support in $\mathbb{R}^{n}$, we have

$$
\left|I_{1} f(x)\right| \leq C\left(M_{\lambda / p} f(x)\right)^{\frac{p}{\lambda}}\left(M_{0} f(x)\right)^{1-\frac{p}{\lambda}},
$$

where $C$ depends only on $n, p$, and $\lambda$.

For $f \not \equiv 0$, let $\delta>0$ to be determined later and set

$$
\begin{aligned}
I_{1} f(x) & =\int_{\mathbb{R}^{n}} f(y)|y-x|^{1-n} d y \\
& =\int_{\{y:|x-y|<\delta\}} f(y)|y-x|^{1-n} d y+\int_{\{y:|x-y| \geq \delta\}} f(y)|y-x|^{1-n} d y \\
& =I+I^{\prime} .
\end{aligned}
$$

Let

$$
a_{k}(x)=\left\{y: 2^{k} \delta \leq|x-y|<2^{k+1} \delta\right\} \quad \text { for } k \in \mathbb{Z}
$$

Then,

$$
\begin{aligned}
|I| & \leq \sum_{k=1}^{\infty} \int_{a_{-k}(x)}|f(y)||x-y|^{1-n} d y \\
& \leq \sum_{k=1}^{\infty}\left(2^{-k} \delta\right)^{1-n}\left(2^{-k+1} \delta\right)^{n} M_{0} f(x)=2^{n} \delta M_{0} f(x) .
\end{aligned}
$$

Similarly,

$$
\begin{aligned}
\left|I^{\prime}\right| & \leq \sum_{k=0}^{\infty} \int_{a_{k}(x)}|f(y)||x-y|^{1-n} d y \\
& \leq \sum_{k=0}^{\infty}\left(2^{k} \delta\right)^{1-n}\left(2^{k+1} \delta\right)^{n-\frac{\lambda}{p}} M_{\lambda / p} f(x)=C \delta^{1-\frac{\lambda}{p}} M_{\lambda / p} f(x),
\end{aligned}
$$


since $p<\lambda$. The choice

$$
\delta=\delta(x)=\left(\frac{M_{\lambda / p} f(x)}{M_{0} f(x)}\right)^{\frac{p}{\lambda}}
$$

finally gives (4.3).

\section{Proof of Theorem 1.3 in the CASE When $\lambda$ IS AN INTEGer}

In this section we prove Theorem 1.3 when $\lambda$ is an integer. The argument is very simple. The case $\lambda \notin \mathbb{Z}$ is the core of our paper and will be considered in Section 6 .

As mentioned in the introduction, the choice of the counterexample when $\lambda \in \mathbb{Z}$ was hinted by the number $p_{1}=\lambda p /(\lambda-p)$, which can be thought of as the Sobolev exponent in dimension $\lambda$. Then, it is natural to choose a function that provides a counterexample for the Sobolev inequality in dimension $\lambda$ and then look at this function embedded in the $n$-dimensional space. Namely, we take

$$
u(x)=\left(\left|x^{\prime}\right|^{-\alpha}-2^{\alpha}\right)_{+} \xi\left(\left|x^{\prime \prime}\right|\right),
$$

where $x=\left(x^{\prime}, x^{\prime \prime}\right) \in \mathbb{R}^{\lambda} \times \mathbb{R}^{n-\lambda}$ and $\xi: \mathbb{R}^{+} \rightarrow[0,1]$ is a cutoff function with $\xi \equiv 1$ in $[0,1 / 2)$ and $\xi \equiv 0$ in $\mathbb{R}^{+} \backslash[0, \sqrt{3} / 3)$. Note that clearly $u$ has support in $B_{1}(0) \subset \mathbb{R}^{n}$.

The rest of the section is devoted to show the following result, which proves Theorem 1.3 when $\lambda$ is an integer.

Proposition 5.1. Let $\lambda$ be an integer such that $1<p<\lambda<n$ and assume that $q>p_{1}:=\lambda p /(\lambda-p)$. Then, for $u$ given by (5.1), we have that $\|u\|_{L^{q}\left(B_{1}\right)}=\infty$ and $\|\nabla u\|_{p, \lambda ; B_{1}}<\infty$ if

$$
\frac{\lambda}{q} \leq \alpha<\frac{\lambda-p}{p}
$$

This proves the optimality of the range $q \leq p_{1}$ for (1.5) when $\lambda \in \mathbb{Z}$, and in turn also for (1.1).

Proof. For every $y \in \bar{B}_{1}^{(n)}$ we have that

$$
\begin{aligned}
\int_{B_{1}^{(n)}} \mid & \left.\nabla u(x)\right|^{p}|x-y|^{\lambda-n} d x \\
& \leq C \int_{B_{1}^{(\lambda)}} \int_{B_{1}^{(n-\lambda)}}\left|x^{\prime}\right|^{-\alpha p-p}|x-y|^{\lambda-n} d x^{\prime \prime} d x^{\prime} \\
& =C \int_{B_{1}^{(\lambda)}}\left|x^{\prime}\right|^{-\alpha p-p} \int_{B_{1}^{(n-\lambda)}}\left(1+\left(\frac{\left|x^{\prime \prime}-y^{\prime \prime}\right|}{\left|x^{\prime}-y^{\prime}\right|}\right)^{2}\right)^{\frac{\lambda-n}{2}}\left|x^{\prime}-y^{\prime}\right|^{\lambda-n} d x^{\prime \prime} d x^{\prime},
\end{aligned}
$$


for some constant $C$ independent of $y$. The change of variables $z=\frac{x^{\prime \prime}-y^{\prime \prime}}{\left|x^{\prime}-y^{\prime}\right|}$ yields

$$
\begin{aligned}
\int_{B_{1}^{(n-\lambda)}} & \left(1+\left(\frac{\left|x^{\prime \prime}-y^{\prime \prime}\right|}{\left|x^{\prime}-y^{\prime}\right|}\right)^{2}\right)^{\frac{\lambda-n}{2}}\left|x^{\prime}-y^{\prime}\right|^{\lambda-n} d x^{\prime \prime} \\
& \leq \int_{B_{2 /\left|x^{\prime}-y^{\prime}\right|}^{(n-\lambda)}}\left(1+|z|^{2}\right)^{\frac{\lambda-n}{2}} d z=C \int_{0}^{\frac{2}{\left|x^{\prime}-y^{\prime}\right|}}\left(1+r^{2}\right)^{\frac{\lambda-n}{2}} r^{n-\lambda-1} d r \\
& \leq C \int_{0}^{\frac{2}{\left|x^{\prime}-y^{\prime}\right|}} \max \{1, r\}^{\lambda-n} r^{n-\lambda-1} d r \leq C\left(1+|\log | x^{\prime}-y^{\prime}||\right) .
\end{aligned}
$$

Therefore, we have

$$
\int_{B_{1}^{(n)}}|\nabla u(x)|^{p}|x-y|^{\lambda-n} d x \leq C \int_{B_{1}^{(\lambda)}}\left|x^{\prime}\right|^{-\alpha p-p}\left(1+|\log | x^{\prime}-y^{\prime}||\right) d x^{\prime} .
$$

We claim that the last integral is bounded uniformly in $y^{\prime} \in \bar{B}_{1}^{(\lambda)}$. To verify this, since $|\log | x^{\prime}-y^{\prime}|| \leq \log 2$ for $x^{\prime} \in B_{1}^{(\lambda)} \backslash B_{1}\left(y^{\prime}\right)$ and $\lambda-\alpha p-p>0$, it suffices to control the integral over $B_{1}\left(y^{\prime}\right)$. But then, calling $z:=y^{\prime}-x^{\prime}$, the integral becomes $\int_{B_{1}^{(\lambda)}} h(z)\left|z-y^{\prime}\right|^{-\alpha p-p} d z$ with $h(z)=1+|\log | z||=1-\log |z|$ for $z \in B_{1}^{(\lambda)}$. Now, since $h$ is non-negative and radially decreasing in $B_{1}^{(\lambda)}$, we can apply Lemma 2.1 with $\eta=0$ and conclude that the largest value of the integral corresponds to $y^{\prime}=0$. But since we have assumed $\lambda-\alpha p-p>0$, the integral with $y^{\prime}=0$ is finite.

On the other hand,

$$
\begin{aligned}
\|u\|_{L^{q}\left(B_{1}\right)}^{q} & \geq \int_{B_{1 / 2}^{(\lambda)}} \int_{B_{1 / 2}^{(n-\lambda)}} u^{q} d x^{\prime \prime} d x^{\prime} \\
& =C \int_{B_{1 / 2}^{(\lambda)}}\left(\left|x^{\prime}\right|^{-\alpha}-2^{\alpha}\right)^{q} d x^{\prime}=C \int_{0}^{\frac{1}{2}}\left(r^{-\alpha}-2^{\alpha}\right)^{q} r^{\lambda-1} d r
\end{aligned}
$$

and the last integral is divergent since $\lambda \leq \alpha q$ by hypothesis.

\section{Proof of Theorem 1.3 in the general CASE}

In this section we conclude the proof of Theorem 1.3 by considering the case when $\lambda$ is not an integer.

Let us motivate first the case $n-1<\lambda<n$. As we have seen in Section 5, when $\lambda$ is equal to $n-1$ expression (5.1) provides a counterexample to the embedding (1.5) when $q>p_{1}$, and therefore also to (1.1) in view of (1.4). On the other hand, when $\lambda$ is equal to $n$ the Morrey and triple norms coincide with the Sobolev norm and $u(x)=\left(|x|^{-\alpha}-2^{\alpha}\right)_{+}$provides a counterexample to embedding (1.5) for $q>p_{1}=p^{*}$ (in this case (1.5) is simply the Sobolev embedding). In both cases the function that yields the counterexample is basically a negative power of the distance function, either to the origin in the case $\lambda=n$, or to a line when $\lambda=n-1$. Therefore, when $\lambda$ is strictly between $n-1$ and $n$, a negative power of the distance to a fractal set of non-integer dimension $n-\lambda$ is a natural candidate to be a counterexample to inequality (1.5). 


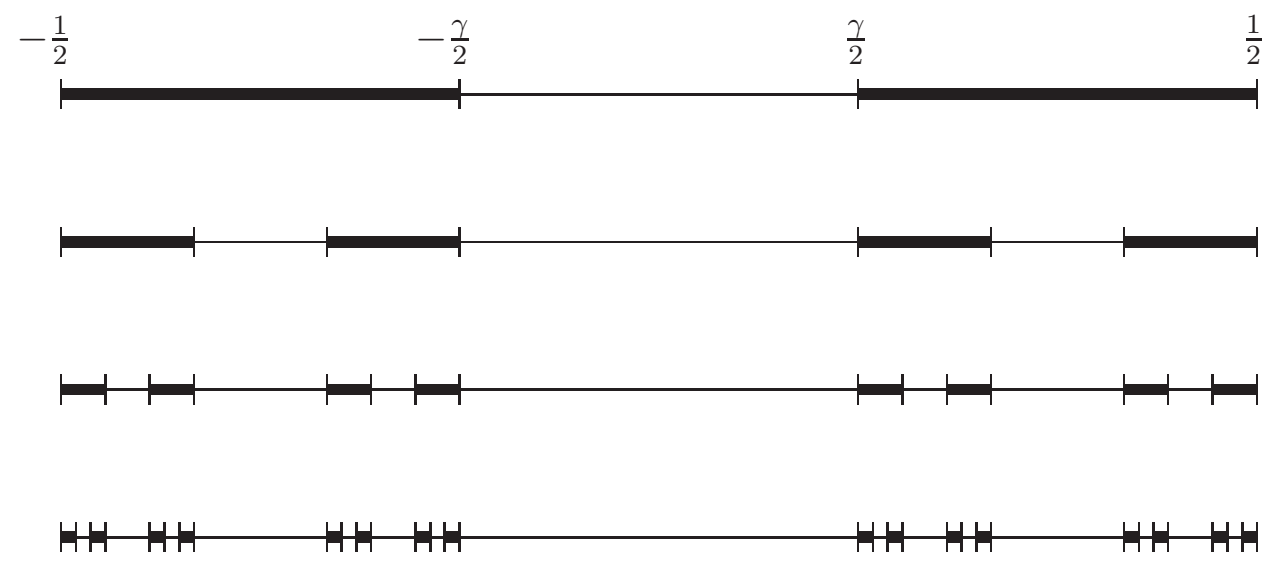

Figure 2. Construction of the generalized Cantor set $C_{\gamma}$

Let us describe precisely the functions that provide the counterexample. When $n-1<\lambda<n$, we consider

$$
u_{\alpha, n}(x)=\left(\operatorname{dist}\left(x, \mathcal{C}_{n, \lambda}\right)^{-\alpha}-4^{\alpha}\right)_{+}
$$

for $\mathcal{C}_{n, \lambda}=\{0\} \times C_{\gamma} \subset \mathbb{R}^{n-1} \times[-1 / 2,1 / 2]$, where $C_{\gamma}$ is a generalized Cantor set with parameter

$$
\gamma=1-2^{1-\frac{1}{n-\lambda}} \in(0,1)
$$

The generalized Cantor set $C_{\gamma}$ (see [9]) is obtained from the interval $[-1 / 2,1 / 2]$ by removing at iteration $j=1,2, \ldots$ the central interval of length $\gamma l_{j-1}$ from each remaining segment of length $l_{j-1}=((1-\gamma) / 2)^{j-1}$; see Figure 2, A precise expression for $C_{\gamma}$ is given later in (6.8), (6.9), and (6.10). The usual Cantor set corresponds to $\gamma=1 / 3$.

The reason for our choice of $\gamma$ in (6.2) is that the Hausdorff dimension of $C_{\gamma}$ is

$$
\frac{-\log 2}{\log \frac{1-\gamma}{2}}=n-\lambda
$$

(see [9, Theorem 9.3]). Thus, letting $\lambda$ vary between $n-1$ and $n$ yields any fractal dimension between 0 and 1. In particular, (6.1) somehow interpolates the integer cases $\lambda=n-1$ and $\lambda=n$.

Note that $u_{\alpha, n}$ has support in $B_{3 / 4}^{(n)}(0)$. Indeed, if $y \in \mathcal{C}_{n, \lambda}$ then $|y| \leq 1 / 2$, and thus $|x-y| \geq 1 / 4$ if $|x| \geq 3 / 4$; in particular $\operatorname{dist}\left(x, \mathcal{C}_{n, \lambda}\right) \geq 1 / 4$ and $u_{\alpha, n}(x)=0$.

In the case when $k-1<\lambda<k$ for some integer $k \in\{2, \ldots, n-1\}$ we embed into $\mathbb{R}^{n}$ the counterexample in $\mathbb{R}^{k}$ by means of an appropriate cutoff function (as we did in the previous section when $\lambda \in \mathbb{Z}$ ). In this way, we reduce the proof to the case $n-1<\lambda<n$. More precisely, we consider

$$
u_{\alpha}(x)=u_{\alpha, k}\left(x^{\prime}\right) \xi\left(\left|x^{\prime \prime}\right|\right) \quad \text { if } k<n,
$$


where $x=\left(x^{\prime}, x^{\prime \prime}\right) \in \mathbb{R}^{k} \times \mathbb{R}^{n-k}, u_{\alpha, k}$ is given by (6.1) with $n$ replaced by $k$, i.e.,

$$
u_{\alpha, k}\left(x^{\prime}\right)=\left(\operatorname{dist}\left(x^{\prime}, \mathcal{C}_{k, \lambda}\right)^{-\alpha}-4^{\alpha}\right)_{+},
$$

and $\xi: \mathbb{R}^{+} \rightarrow[0,1]$ is a cutoff function with $\xi \equiv 1$ in $[0,1 / 2)$ and $\xi \equiv 0$ in $\mathbb{R}^{+} \backslash[0, \sqrt{3} / 3)$. Note that if $u_{\alpha}(x) \neq 0$ then necessarily $\left|x^{\prime}\right| \leq 3 / 4$ and $\left|x^{\prime \prime}\right| \leq \sqrt{3} / 3$; thus $|x|<1$.

The rest of the section is devoted to proving the following result, which together with Proposition 5.1 completes the proof of Theorem 1.3 .

Theorem 6.1. Let $p, \lambda, q \in \mathbb{R}$ be such that $1<p<\lambda<n, k-1<\lambda<k$ for some integer $k \in\{2, \ldots, n\}$, and $q \geq 1$. Consider

$$
u_{\alpha}(x)= \begin{cases}\left(\operatorname{dist}\left(x, \mathcal{C}_{n, \lambda}\right)^{-\alpha}-4^{\alpha}\right)_{+} & \text {if } k=n \\ \left(\operatorname{dist}\left(x^{\prime}, \mathcal{C}_{k, \lambda}\right)^{-\alpha}-4^{\alpha}\right)_{+} \xi\left(\left|x^{\prime \prime}\right|\right) & \text { if } k \in\{2, \ldots, n-1\},\end{cases}
$$

with $x=\left(x^{\prime}, x^{\prime \prime}\right) \in \mathbb{R}^{k} \times \mathbb{R}^{n-k}, \xi: \mathbb{R}^{+} \rightarrow[0,1]$ a cutoff function as described after (6.4), and $\mathcal{C}_{k, \lambda}$ a set of Hausdorff dimension $k-\lambda$ given by

$$
\mathcal{C}_{k, \lambda}=\{0\} \times C_{\gamma} \subset \mathbb{R}^{k-1} \times[-1 / 2,1 / 2],
$$

where $C_{\gamma} \subset[-1 / 2,1 / 2]$ is a generalized Cantor set with parameter $\gamma=1-2^{1-\frac{1}{k-\lambda}}$. Then,

$$
\left\|u_{\alpha}\right\|_{L^{q}\left(B_{1}^{(n)}\right)}=\infty \quad \text { and } \quad\left\|\nabla u_{\alpha}\right\|_{p, \lambda ; B_{1}^{(n)}}<\infty
$$

if

$$
\frac{\lambda}{q} \leq \alpha<\frac{\lambda-p}{p}
$$

This proves the optimality of the range $q \leq p_{1}=\lambda p /(\lambda-p)$ for inequality (1.5), and in turn also for (1.1).

The proof of Theorem 6.1 is divided into two parts. In the first part we reduce the computations from dimension $n$ to dimension $k$ with a similar argument to the one in the proof of Proposition 5.1. More precisely we will show, for $u_{\alpha, k}$ given by (6.4), that

$$
\left\|\nabla u_{\alpha}\right\|_{p, \lambda ; B_{1}^{(n)}} \leq C\left\|\nabla u_{\alpha, k} \mid\right\|_{p, \lambda ; B_{1}^{(k)}}
$$

and

$$
\left\|u_{\alpha}\right\|_{L^{q}\left(B_{1}^{(n)}\right)} \geq C^{-1}\left\|u_{\alpha, k}\right\|_{L^{q}\left(B_{1}^{(k)}\right)},
$$

for some constant $C$, and hence that it is enough to study the case $k=n$ taking $u_{\alpha}=u_{\alpha, n}$. In the second part of the proof we will show that (6.5) leads to

$$
\left\|u_{\alpha, n}\right\|_{L^{q}\left(B_{1}^{(n)}\right)}=\infty \quad \text { and } \quad\left\|\nabla u_{\alpha, n}\right\| \|_{p, \lambda ; B_{1}^{(n)}}<\infty
$$

as desired; this part is the content of the following two propositions.

Proposition 6.2. Let $\lambda, q \in \mathbb{R}$ be such that $\lambda>n-1$ and $q \geq 1$. Consider $u_{\alpha, n}$ given by (6.1) with $\alpha$ such that $\lambda \leq \alpha q$. Then, $\left\|u_{\alpha, n}\right\|_{L^{q\left(B_{1}^{(n)}\right)}}=\infty$. 
Proposition 6.3. Let $\lambda, p \in \mathbb{R}$ be such that $n-1<\lambda<n, p>1$ and consider $u_{\alpha, n}$ given by (6.1) with $\alpha>0$ satisfying

$$
\alpha<\frac{\lambda-p}{p} .
$$

Then,

$$
\left\|\left|\nabla u_{\alpha, n}\right|\right\|_{p, \lambda ; B_{1}^{(n)}}^{p}:=\sup _{y \in \bar{B}_{1}^{(n)}} \int_{B_{1}^{(n)}}\left|\nabla u_{\alpha, n}(x)\right|^{p}|x-y|^{\lambda-n} d x<\infty .
$$

Let us now prove Theorem 6.1 assuming Propositions 6.2 and 6.3, which will be established afterwards.

Proof of Theorem 6.1. Since Propositions 6.2 and 6.3 yield the result when $k=n$, let us assume $k<n$. Let $y \in \bar{B}_{1}^{(n)}$. By (6.3) and (6.4) we have

$$
\left|\nabla u_{\alpha}(x)\right| \leq C\left(\left|\nabla u_{\alpha, k}\left(x^{\prime}\right)\right|+u_{\alpha, k}\left(x^{\prime}\right)\right) \leq C\left|\nabla u_{\alpha, k}\left(x^{\prime}\right)\right|
$$

for almost every $x \in B_{1}^{(n)}$, where we have used that the modulus of the gradient of a distance function is equal to 1 a.e. Therefore,

$$
\begin{aligned}
& \int_{B_{1}^{(n)}}\left|\nabla u_{\alpha}(x)\right|^{p}|x-y|^{\lambda-n} d x \leq C \int_{B_{1}^{(k)}} \int_{B_{1}^{(n-k)}}\left|\nabla u_{\alpha, k}\left(x^{\prime}\right)\right|^{p}|x-y|^{\lambda-n} d x^{\prime \prime} d x^{\prime} \\
& =C \int_{B_{1}^{(k)}}\left|\nabla u_{\alpha, k}\left(x^{\prime}\right)\right|^{p}\left|x^{\prime}-y^{\prime}\right|^{\lambda-k} \int_{B_{1}^{(n-k)}}\left(1+\left(\frac{\left|x^{\prime \prime}-y^{\prime \prime}\right|}{\left|x^{\prime}-y^{\prime}\right|}\right)^{2}\right)^{\frac{\lambda-n}{2}}\left|x^{\prime}-y^{\prime}\right|^{k-n} d x^{\prime \prime} d x^{\prime} .
\end{aligned}
$$

The change of variables $z=\frac{x^{\prime \prime}-y^{\prime \prime}}{\left|x^{\prime}-y^{\prime}\right|}$ yields

$$
\begin{aligned}
\int_{B_{1}^{(n-k)}} & \left(1+\left(\frac{\left|x^{\prime \prime}-y^{\prime \prime}\right|}{\left|x^{\prime}-y^{\prime}\right|}\right)^{2}\right)^{\frac{\lambda-n}{2}}\left|x^{\prime}-y^{\prime}\right|^{k-n} d x^{\prime \prime} \leq \int_{B_{2 /\left|x^{\prime}-y^{\prime}\right|}^{(n-k)}}\left(1+|z|^{2}\right)^{\frac{\lambda-n}{2}} d z \\
= & C \int_{0}^{\frac{2}{\left|x^{\prime}-y^{\prime}\right|}}\left(1+r^{2}\right)^{\frac{\lambda-n}{2}} r^{n-k-1} d r \leq C \int_{0}^{\frac{2}{x^{\prime}-y^{\prime} \mid}} \max \{1, r\}^{\lambda-n} r^{n-k-1} d r \\
= & C\left(\int_{0}^{1} r^{n-k-1} d r+\int_{1}^{\frac{2}{\left|x^{\prime}-y^{\prime}\right|}} r^{\lambda-k-1} d r\right) \leq C
\end{aligned}
$$

independently of $y$. Therefore,

$$
\begin{aligned}
\int_{B_{1}^{(n)}} & \left|\nabla u_{\alpha}(x)\right|^{p}|x-y|^{\lambda-n} d x \\
& \leq C \sup _{y^{\prime} \in \bar{B}_{1}^{(k)}} \int_{B_{1}^{(k)}}\left|\nabla u_{\alpha, k}\left(x^{\prime}\right)\right|^{p}\left|x^{\prime}-y^{\prime}\right|^{\lambda-k} d x^{\prime}=C\|\| \nabla u_{\alpha, k} \mid \|_{p, \lambda ; B_{1}^{(k)}},
\end{aligned}
$$

and Proposition 6.3 applied in $\mathbb{R}^{k}$, i.e., with $n$ replaced by $k$, yields $\left\|\left|\nabla u_{\alpha}\right|\right\|_{p, \lambda ; B_{1}^{(n)}}<\infty$.

On the other hand,

$$
\left\|u_{\alpha}\right\|_{L^{q}\left(B_{1}^{(n)}\right)}^{q}=\int_{B_{3 / 4}^{(k)}} u_{\alpha, k}^{q}\left(x^{\prime}\right) \int_{B_{\sqrt{3} / 3}^{(n-k)}} \xi^{q}\left(\left|x^{\prime \prime}\right|\right) d x^{\prime \prime} d x^{\prime}=C\left\|u_{\alpha, k}\right\|_{L^{q}\left(B_{3 / 4}^{(k)}\right)}^{q},
$$


which is infinite by Proposition 6.2 applied with $n=k$, since (as we pointed out) $u_{\alpha, k}$ given by (6.4) has support in $\bar{B}_{3 / 4}^{(k)}$.

We devote the rest of the section to the proofs of Propositions 6.2 and 6.3. In the sequel we assume

$$
n-1<\lambda<n \text {. }
$$

Recall that

$$
u_{\alpha, n}(x)=\left(\operatorname{dist}\left(x, \mathcal{C}_{n, \lambda}\right)^{-\alpha}-4^{\alpha}\right)_{+}
$$

for $\mathcal{C}_{n, \lambda}=\{0\} \times C_{\gamma} \subset \mathbb{R}^{n-1} \times[-1 / 2,1 / 2]$, where $C_{\gamma}$ is the generalized Cantor set with parameter $\gamma=1-2^{1-\frac{1}{n-\lambda}}$ defined in the beginning of this section. We have

$$
\left[-\frac{1}{2}, \frac{1}{2}\right] \backslash C_{\gamma}:=\bigcup_{l=1}^{\infty} \bigcup_{m=1}^{2^{l-1}} G_{l, m}
$$

where the union is disjoint and $G_{l, m}$ are the $2^{l-1}$ gap-intervals introduced in generation $l$, namely 2

$$
\begin{aligned}
G_{l, m} & =\left(\frac{1-\gamma}{2}\right)^{l-1}\left(-\frac{\gamma}{2}, \frac{\gamma}{2}\right)+h_{l, m} \\
& =\left(h_{l, m}-\frac{\gamma}{2}\left(\frac{1-\gamma}{2}\right)^{l-1}, h_{l, m}+\frac{\gamma}{2}\left(\frac{1-\gamma}{2}\right)^{l-1}\right),
\end{aligned}
$$

where

$$
h_{l, m}=-\frac{1}{2}+\frac{1}{2}\left(\frac{1-\gamma}{2}\right)^{l-1}+\frac{1+\gamma}{2} \sum_{j=1}^{l-1} c_{j}\left(\frac{1-\gamma}{2}\right)^{j-1} .
$$

Here $c_{j} \in\{0,1\}$ for all $j=1, \ldots, l-1$, and the index $m \in\left\{1,2, \ldots, 2^{l-1}\right\}$ runs through all possible choices of the coefficients $c_{1} c_{2} \ldots c_{l-1}$; see Figure 2 above.

As a consequence of (6.8), we have that $C_{\gamma}$ is a compact set of Lebesgue measure 0. In addition, the set $C_{\gamma}$ is self-similar, that is, $C_{\gamma}=S_{1}\left(C_{\gamma}\right) \cup S_{2}\left(C_{\gamma}\right)$ where $S_{1}(t)=(1-\gamma) t / 2-(1+\gamma) / 4$ and $S_{2}(t)=(1-\gamma) t / 2+(1+\gamma) / 4$. Finally, the Hausdorff dimension of $C_{\gamma}$ is $\left.-\log 2 / \log ((1-\gamma) / 2)\right)$, see [9, Theorem 9.3]. Notice that we have chosen $\gamma=1-2^{1-\frac{1}{n-\lambda}}$ such that the Hausdorff dimension of $C_{\gamma}$ is $n-\lambda$.

6.1. Proof of Proposition 6.2: Computation of the $L^{q}$ norm. In this subsection we provide the proof of Proposition 6.2

\footnotetext{
${ }^{2}$ We will not need the following precise expression for the gaps, but only to understand their size and self-similar structure.
} 
Proof of Proposition 6.2. Recall that $\mathcal{C}_{n, \lambda}=\{0\} \times C_{\gamma} \subset \mathbb{R}^{n-1} \times[-1 / 2,1 / 2]$. We will denote $x=\left(x^{\prime}, x^{\prime \prime}\right) \in \mathbb{R}^{n-1} \times \mathbb{R}$. Since $C_{\gamma}$ has zero Lebesgue measure, (6.8) leads to

$$
\begin{aligned}
\left\|u_{\alpha, n}\right\|_{L^{q}\left(B_{1}^{(n)}\right)}^{q} & \geq \int_{-\frac{1}{2}}^{\frac{1}{2}} \int_{B_{1 / 4}^{(n-1)}}\left(\operatorname{dist}\left(x, \mathcal{C}_{n, \lambda}\right)^{-\alpha}-4^{\alpha}\right)_{+}^{q} d x^{\prime} d x^{\prime \prime} \\
& =\sum_{l=1}^{\infty} \sum_{m=1}^{2^{l-1}} \int_{G_{l, m}} \int_{B_{1 / 4}^{(n-1)}}\left(\operatorname{dist}\left(x, \mathcal{C}_{n, \lambda}\right)^{-\alpha}-4^{\alpha}\right)_{+}^{q} d x^{\prime} d x^{\prime \prime}
\end{aligned}
$$

where $G_{l, m}$ are given by (6.9) and (6.10).

An affine change of variables $x^{\prime}=\left(\frac{1-\gamma}{2}\right)^{l-1} t^{\prime}, x^{\prime \prime}=\left(\frac{1-\gamma}{2}\right)^{l-1} t^{\prime \prime}+h_{l, m}$ and the selfsimilarity of $\mathcal{C}_{n, \lambda}$ yield

$$
\begin{aligned}
& \int_{G_{l, m}} \int_{B_{1 / 4}^{(n-1)}}\left(\operatorname{dist}\left(x, \mathcal{C}_{n, \lambda}\right)^{-\alpha}-4^{\alpha}\right)_{+}^{q} d x^{\prime} d x^{\prime \prime} \\
& =\left(\frac{1-\gamma}{2}\right)^{(n-\alpha q)(l-1)} \int_{-\frac{\gamma}{2}}^{\frac{\gamma}{2}} \int_{\left\{\left|t^{\prime}\right| \leq \frac{1}{4}\left(\frac{2}{1-\gamma}\right)^{l-1}\right\}}\left(\operatorname{dist}\left(t, \mathcal{C}_{n, \lambda}\right)^{-\alpha}-\left(\frac{1-\gamma}{2}\right)^{\alpha(l-1)} 4^{\alpha}\right)_{+}^{q} d t^{\prime} d t^{\prime \prime} \\
& \geq\left(\frac{1-\gamma}{2}\right)^{(n-\alpha q)(l-1)} \int_{-\frac{\gamma}{2}}^{\frac{\gamma}{2}} \int_{B_{1 / 4}^{(n-1)}}\left(\operatorname{dist}\left(t, \mathcal{C}_{n, \lambda}\right)^{-\alpha}-4^{\alpha}\right)_{+}^{q} d t^{\prime} d t^{\prime \prime}
\end{aligned}
$$

for each $m \in\left\{1, \ldots, 2^{l-1}\right\}$. Therefore, adding these $2^{l-1}$ integrals of generation $l$, and then summing in $l$, we have

$$
\left\|u_{\alpha, n}\right\|_{L^{q\left(B_{1}^{(n)}\right)}}^{q} \geq \sum_{l=1}^{\infty}\left(2\left(\frac{1-\gamma}{2}\right)^{n-\alpha q}\right)^{l-1} \int_{-\frac{\gamma}{2}}^{\frac{\gamma}{2}} \int_{B_{1 / 4}^{(n-1)}}\left(\operatorname{dist}\left(t, \mathcal{C}_{n, \lambda}\right)^{-\alpha}-4^{\alpha}\right)_{+}^{q} d t^{\prime} d t^{\prime \prime} .
$$

Since the integral on the right-hand side is positive, it is enough to show that the series diverges. This happens whenever

$$
2\left(\frac{1-\gamma}{2}\right)^{n-\alpha q} \geq 1
$$

which by our choice of $\gamma$ is equivalent to

$$
n-\alpha q \leq \frac{-\log 2}{\log \frac{1-\gamma}{2}}=n-\lambda .
$$

This inequality holds by hypothesis.

6.2. Proof of Proposition 6.3: Bound for the "triple norm". The proof of Proposition 6.3 has two parts. The first one (Lemma 6.6 below) shows that in order to bound the triple norm of $\nabla u_{\alpha, n}$, it suffices to only consider points $y \in\{0\} \times[-1 / 2,1 / 2]$, instead of the full ${\overline{B_{1}}}^{(n)}$. More precisely, we prove that

$$
\left\|\nabla u_{\alpha, n}\right\|_{p, \lambda ; B_{1}^{(n)}}^{p} \leq C \sup _{y^{\prime}=0,\left|y^{\prime \prime}\right| \leq \frac{1}{2}} \int_{-\frac{1}{2}}^{\frac{1}{2}} \int_{B_{1 / 4}^{(n-1)}} \operatorname{dist}\left(x, \mathcal{C}_{n, \lambda}\right)^{-\alpha p-p}|x-y|^{\lambda-n} d x^{\prime} d x^{\prime \prime} .
$$


Since $C_{\gamma}$ has zero Lebesgue measure, by (6.8) we can write the outer integral on the right-hand side of (6.11) as an infinite sum of integrals over the disjoint gap-intervals $G_{l, m}$ of decreasing size.

The second part of the proof of Proposition 6.3, and crucial point in the argument, is how to estimate these integrals in terms of the size of the gaps in such a way that the series converges. Here, there are two cases to be considered according to the position of the singularity $y$ relative to a given gap: the case when $y$ lies on the closure of a gap (and hence the function $x \mapsto|x-y|^{\lambda-n}$ is singular), and the case when the gap is uniformly away from $y$ (and hence $|x-y|^{\lambda-n}$ can be bounded above and factored out from the integral). We deal with these two cases in Lemmas 6.4 and 6.5 respectively.

Lemma 6.4. Let $G=((1-\gamma) / 2)^{l-1}(-\gamma / 2, \gamma / 2)+h$ for some $l \geq 1$ and $h \in \mathbb{R}$ of the form (6.10) (i.e. $G$ is a gap-interval introduced in generation l). Assume $y \in\{0\} \times \bar{G}$ and $\lambda-\alpha p-p>0$. Then, we have that

$$
\begin{aligned}
& \int_{G} \int_{B_{1 / 4}^{(n-1)}} \operatorname{dist}\left(x, \mathcal{C}_{n, \lambda}\right)^{-\alpha p-p}|x-y|^{\lambda-n} d x^{\prime} d x^{\prime \prime} \\
& \leq C\left(\left(\frac{1-\gamma}{2}\right)^{(\lambda-\alpha p-p)(l-1)}+l\left(\frac{1-\gamma}{2}\right)^{l-1}\right)
\end{aligned}
$$

for a constant $C$ depending only on $n, p, \lambda$, and $\alpha$.

Proof. Denote $a=(\gamma / 2)((1-\gamma) / 2)^{l-1}$ so that $G=(-a, a)+h$. To relate $\operatorname{dist}\left(x, \mathcal{C}_{n, \lambda}\right)$ and $|x-y|$ we consider the midpoints between $y^{\prime \prime}$ and $h+a$, and between $y^{\prime \prime}$ and $h-a$. In this way, we have the bound

$$
\begin{aligned}
\int_{G} \int_{B_{1 / 4}^{(n-1)}} \operatorname{dist}\left(x, \mathcal{C}_{n, \lambda}\right)^{-\alpha p-p}|x-y|^{\lambda-n} d x^{\prime} d x^{\prime \prime} \\
\leq \int_{\frac{y^{\prime \prime}+h+a}{2}}^{h+a} \int_{B_{1 / 4}^{(n-1)}} \operatorname{dist}\left(x, \mathcal{C}_{n, \lambda}\right)^{\lambda-n-\alpha p-p} d x^{\prime} d x^{\prime \prime} \\
\quad+\int_{\frac{y^{\prime \prime}+h-a}{2}}^{\frac{y^{\prime \prime}+h+a}{2}} \int_{B_{1 / 4}^{(n-1)}}|x-y|^{\lambda-n-\alpha p-p} d x^{\prime} d x^{\prime \prime} \\
+\int_{h-a}^{\frac{y^{\prime \prime}+h-a}{2}} \int_{B_{1 / 4}^{(n-1)}} \operatorname{dist}\left(x, \mathcal{C}_{n, \lambda}\right)^{\lambda-n-\alpha p-p} d x^{\prime} d x^{\prime \prime}=I_{1}+I_{2}+I_{3} .
\end{aligned}
$$

Let us estimate $I_{1}$ first. For this, notice that whenever $x^{\prime \prime} \in G$, we have

$$
\operatorname{dist}\left(x, \mathcal{C}_{n, \lambda}\right)^{2}=\left|x^{\prime}\right|^{2}+\min \left\{\left(h+a-x^{\prime \prime}\right)^{2},\left(x^{\prime \prime}-h+a\right)^{2}\right\} .
$$

Therefore,

$$
I_{1}=\int_{\frac{y^{\prime \prime}+h+a}{2}}^{h+a} \int_{B_{1 / 4}^{(n-1)}}\left(\left|x^{\prime}\right|^{2}+\left(h+a-x^{\prime \prime}\right)^{2}\right)^{\frac{\lambda-n-\alpha p-p}{2}} d x^{\prime} d x^{\prime \prime}
$$


and a change to cylindrical coordinates and the change of variables $t=h+a-x^{\prime \prime}$ yield

$$
I_{1}=C \int_{0}^{\frac{h+a-y^{\prime \prime}}{2}} \int_{0}^{\frac{1}{4}}\left(r^{2}+t^{2}\right)^{\frac{\lambda-n-\alpha p-p}{2}} r^{n-2} d r d t .
$$

Similarly,

$$
I_{3}=C \int_{\frac{h-a-y^{\prime \prime}}{2}}^{0} \int_{0}^{\frac{1}{4}}\left(r^{2}+t^{2}\right)^{\frac{\lambda-n-\alpha p-p}{2}} r^{n-2} d r d t .
$$

On the other hand, since $y^{\prime}=0$, we have

$$
\begin{aligned}
I_{2} & =\int_{\frac{y^{\prime \prime}+h-a}{2}}^{\frac{y^{\prime \prime}+h+a}{2}} \int_{B_{1 / 4}^{(n-1)}}\left(\left|x^{\prime}\right|^{2}+\left(x^{\prime \prime}-y^{\prime \prime}\right)^{2}\right)^{\frac{\lambda-n-\alpha p-p}{2}} d x^{\prime} d x^{\prime \prime} \\
& =C \int_{\frac{y^{\prime \prime}+h-a}{2}}^{\frac{y^{\prime \prime}+h+a}{2}} \int_{0}^{\frac{1}{4}}\left(r^{2}+\left(x^{\prime \prime}-y^{\prime \prime}\right)^{2}\right)^{\frac{\lambda-n-\alpha p-p}{2}} r^{n-2} d r d x^{\prime \prime} \\
& =C \int_{\frac{h-a-y^{\prime \prime}}{2}}^{\frac{h+a-y^{\prime \prime}}{2}} \int_{0}^{\frac{1}{4}}\left(r^{2}+t^{2}\right)^{\frac{\lambda-n-\alpha p-p}{2}} r^{n-2} d r d t,
\end{aligned}
$$

after applying a change to cylindrical coordinates and the change of variables $t=$ $x^{\prime \prime}-y^{\prime \prime}$.

Then, (6.13) and (6.15) - (6.17), and the change of variables $z_{1}=2 t-h+y^{\prime \prime}, z_{2}=2 r$ give

$$
\begin{aligned}
\int_{G} \int_{B_{1 / 4}^{(n-1)}} \operatorname{dist}\left(x, \mathcal{C}_{n, \lambda}\right)^{-\alpha p-p}|x-y|^{\lambda-n} d x^{\prime} d x^{\prime \prime} \\
\leq 2 C \int_{\frac{h-a-y^{\prime \prime}}{2}}^{\frac{h+a-y^{\prime \prime}}{2}} \int_{0}^{\frac{1}{4}}\left(r^{2}+t^{2}\right)^{\frac{\lambda-n-\alpha p-p}{2}} r^{n-2} d r d t \\
=C 2^{1-\lambda+\alpha p+p} \int_{-a}^{a} \int_{0}^{\frac{1}{2}}\left(\left(z_{1}+h-y^{\prime \prime}\right)^{2}+z_{2}^{2}\right)^{\frac{\lambda-n-\alpha p-p}{2}} z_{2}^{n-2} d z_{2} d z_{1} \\
=C 2^{1-\lambda+\alpha p+p} J\left(y^{\prime \prime}-h, 0\right),
\end{aligned}
$$

for

$$
J(y)=\int_{-a}^{a} \int_{0}^{\frac{1}{2}}|z-y|^{\lambda-n-\alpha p-p} z_{2}^{n-2} d z_{2} d z_{1} .
$$

Here we can apply Lemma 2.1 in dimension 2 , with $\Omega=[-a, a] \times[0,1 / 2], \theta=n-\lambda+$ $\alpha p+p, h(z)=z_{2}^{n-2}$, and $\eta=0$. Therefore, $J$ is non-increasing with respect to $y_{1}$ in $[0, \infty)$, and non-decreasing with respect to $y_{1}$ in $(-\infty, 0]$. In particular, $J\left(y^{\prime \prime}-h, 0\right) \leq$ $J(0)$, with an equality for $y^{\prime \prime}=h$. We conclude

$$
\begin{aligned}
& \int_{G} \int_{B_{1 / 4}^{(n-1)}} \operatorname{dist}\left(x, \mathcal{C}_{n, \lambda}\right)^{-\alpha p-p}|x-y|^{\lambda-n} d x^{\prime} d x^{\prime \prime} \\
& \leq C 2^{1-\lambda+\alpha p+p} \int_{-a}^{a} \int_{0}^{\frac{1}{2}}\left(z_{1}^{2}+z_{2}^{2}\right)^{\frac{\lambda-n-\alpha p-p}{2}} z_{2}^{n-2} d z_{2} d z_{1}
\end{aligned}
$$


independently of $y$.

We estimate now the integral on the right-hand side of (6.18) by applying the change of variables $x_{1}=z_{1}, z_{2}=\left|x_{1}\right| x_{2}$

$$
\begin{aligned}
\int_{-a}^{a} \int_{0}^{\frac{1}{2}} & \left(z_{1}^{2}+z_{2}^{2}\right)^{\frac{\lambda-n-\alpha p-p}{2}} z_{2}^{n-2} d z_{2} d z_{1} \\
& =\int_{-a}^{a}\left|x_{1}\right|^{\lambda-\alpha p-p-1} \int_{0}^{\frac{1}{2\left|x_{1}\right|}}\left(1+x_{2}^{2}\right)^{\frac{\lambda-n-\alpha p-p}{2}} x_{2}^{n-2} d x_{2} d x_{1} .
\end{aligned}
$$

Notice that $\left|x_{1}\right|<a<1 / 2$, and therefore we can use $1+x_{2}^{2} \geq \max \left\{1, x_{2}^{2}\right\}$ to show that

$$
\int_{0}^{\frac{1}{2\left|x_{1}\right|}}\left(1+x_{2}^{2}\right)^{\frac{\lambda-n-\alpha p-p}{2}} x_{2}^{n-2} d x_{2} \leq \int_{0}^{1} x_{2}^{n-2} d x_{2}+\int_{1}^{\frac{1}{2\left|x_{1}\right|}} x_{2}^{\lambda-\alpha p-p-2} d x_{2} .
$$

Assume first that $\lambda-\alpha p-p \neq 1$ and recall that $\lambda-\alpha p-p>0$ by hypothesis. Then,

$$
\begin{aligned}
\int_{G} \int_{B_{1 / 4}^{(n-1)}} & \operatorname{dist}\left(x, \mathcal{C}_{n, \lambda}\right)^{-\alpha p-p}|x-y|^{\lambda-n} d x^{\prime} d x^{\prime \prime} \\
& \leq C \int_{0}^{a} x_{1}^{\lambda-\alpha p-p-1}\left(1+x_{1}^{-\lambda+\alpha p+p+1}\right) d x_{1} \leq C\left(a^{\lambda-\alpha p-p}+a\right) .
\end{aligned}
$$

On the other hand, if $\lambda-\alpha p-p=1$, then

$$
\begin{aligned}
& \int_{G} \int_{B_{1 / 4}^{(n-1)}} \operatorname{dist}\left(x, \mathcal{C}_{n, \lambda}\right)^{-\alpha p-p}|x-y|^{\lambda-n} d x^{\prime} d x^{\prime \prime} \\
& \leq C \int_{0}^{a}\left(1-\log 2 x_{1}\right) d x_{1} \leq C(a+a|\log a|) .
\end{aligned}
$$

In both cases,

$$
\int_{G} \int_{B_{1 / 4}^{(n-1)}} \operatorname{dist}\left(x, \mathcal{C}_{n, \lambda}\right)^{-\alpha p-p}|x-y|^{\lambda-n} d x^{\prime} d x^{\prime \prime} \leq C\left(a^{\lambda-\alpha p-p}+a(1+|\log a|)\right)
$$

and the lemma is proved.

The following lemma will allow us to control the triple norm when $y$ belongs to a gap different from $G$. Notice that the exponents on the right-hand side of the estimate are different in Lemmas 6.4 and 6.5 .

Lemma 6.5. Let $G=((1-\gamma) / 2)^{l-1}(-\gamma / 2, \gamma / 2)+h$ for some $l \geq 1$ and $h \in \mathbb{R}$ of the form (6.10) (i.e. $G$ is a gap-interval introduced in generation l). Assume $n-\alpha p-p>0$. Then, we have that

$$
\int_{G} \int_{B_{1 / 4}^{(n-1)}} \operatorname{dist}\left(x, \mathcal{C}_{n, \lambda}\right)^{-\alpha p-p} d x^{\prime} d x^{\prime \prime} \leq C\left(\left(\frac{1-\gamma}{2}\right)^{(n-\alpha p-p)(l-1)}+l\left(\frac{1-\gamma}{2}\right)^{l-1}\right)
$$

for a constant $C$ depending only on $n, p$, and $\alpha$. 
Proof. Let $a=(\gamma / 2)((1-\gamma) / 2)^{l-1}$ so that $G=(-a, a)+h$. Using cylindrical coordinates and (6.14), we have

$$
\begin{aligned}
\int_{G} \int_{B_{1 / 4}^{(n-1)}} \operatorname{dist}\left(x, \mathcal{C}_{n, \lambda}\right)^{-\alpha p-p} d x^{\prime} d x^{\prime \prime} \\
=C\left(\int_{h}^{h+a} \int_{0}^{\frac{1}{4}}\left(r^{2}+\left(h+a-x^{\prime \prime}\right)^{2}\right)^{\frac{-\alpha p-p}{2}} r^{n-2} d r d x^{\prime \prime}\right. \\
\left.\quad+\int_{h-a}^{h} \int_{0}^{\frac{1}{4}}\left(r^{2}+\left(x^{\prime \prime}-h+a\right)^{2}\right)^{\frac{-\alpha p-p}{2}} r^{n-2} d r d x^{\prime \prime}\right) \\
=2 C \int_{0}^{a} \int_{0}^{\frac{1}{4}}\left(r^{2}+\left(z^{\prime \prime}\right)^{2}\right)^{\frac{-\alpha p-p}{2}} r^{n-2} d r d z^{\prime \prime} .
\end{aligned}
$$

Notice that this integral is of the same type as the one in the right-hand side of (6.18), taking $\lambda=n$ there. It is now easy to check that one can proceed as in the final part of Lemma 6.4 (taking $\lambda=n$ there) and complete the proof.

As mentioned before, the following lemma will also be used in the first part of the proof of Proposition 6.3 in order to show that to bound the supremum in the definition of $\left\|\nabla u_{\alpha, n}\right\| \|_{p, \lambda ; B_{1}^{(n)}}$, it suffices to take $y \in\{0\} \times[-1 / 2,1 / 2]$.

Lemma 6.6. Let $u_{\alpha, n}$ be given by (6.7). Then,

$$
\begin{aligned}
\| \nabla u_{\alpha, n}||_{p, \lambda ; B_{1}^{(n)}}^{p} & =\sup _{y \in \bar{B}_{1}^{(n)}} \int_{B_{1}^{(n)}}\left|\nabla u_{\alpha, n}(x)\right|^{p}|x-y|^{\lambda-n} d x \\
& \leq C \sup _{y^{\prime}=0,\left|y^{\prime \prime}\right| \leq \frac{1}{2}} \int_{-\frac{1}{2}}^{\frac{1}{2}} \int_{B_{1 / 4}^{(n-1)}} \operatorname{dist}\left(x, \mathcal{C}_{n, \lambda}\right)^{-\alpha p-p}|x-y|^{\lambda-n} d x^{\prime} d x^{\prime \prime}
\end{aligned}
$$

for some constant $C$ depending only on $n, p$, and $\alpha$.

We postpone the proof of Lemma 6.6 until the end of the section and proceed instead with the proof of Proposition 6.3. The idea is to "cluster" the gaps according to their distance from $y$, and then use Lemmas 6.4 and 6.5.

Proof of Proposition 6.3. As a result of Lemma 6.6 we can assume that $y^{\prime}=0$ and $y^{\prime \prime} \in[-1 / 2,1 / 2]$. To simplify the argument below, by Fatou's lemma we may assume that $y^{\prime \prime}$ is not the midpoint of any gap $G_{l, m}$ given by (6.9) and (6.10).

Each generation $l \geq 1$ introduces $2^{l-1}$ gaps $G_{l, m}$, and we can write

$$
\begin{aligned}
\int_{-\frac{1}{2}}^{\frac{1}{2}} \int_{B_{1 / 4}^{(n-1)}} \operatorname{dist}\left(x, \mathcal{C}_{n, \lambda}\right)^{-\alpha p-p}|x-y|^{\lambda-n} d x^{\prime} d x^{\prime \prime} \\
=\sum_{l=1}^{\infty} \sum_{m=1}^{2^{l-1}} \int_{G_{l, m}} \int_{B_{1 / 4}^{(n-1)}} \operatorname{dist}\left(x, \mathcal{C}_{n, \lambda}\right)^{-\alpha p-p}|x-y|^{\lambda-n} d x^{\prime} d x^{\prime \prime} .
\end{aligned}
$$

Recall that the length of the gap $G_{l, m}$ is $\gamma((1-\gamma) / 2)^{l-1}$. 


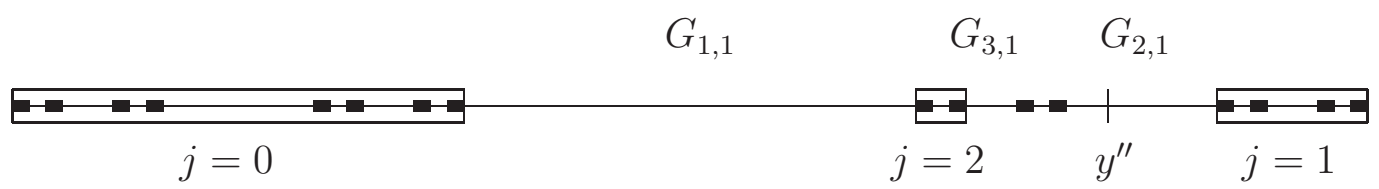

Figure 3. Gaps up to generation $l=4$, classified according to (6.21).

We classify the $2^{l-1}$ gaps of generation $l \geq 2$ according to their distance from $y^{\prime \prime}$ as follows (see Figures 2 and 3 ):

(1) We split $(-1 / 2,1 / 2)$ into two halves, and notice that there are exactly $2^{l-2}$ gaps of generation $l$ in each half. We denote the gaps on the half-interval which does not contain $y^{\prime \prime}$ by $G_{l, m}^{0}$, with $m=1,2, \ldots, 2^{l-2}$. Since half of $G_{1,1}=(-\gamma / 2, \gamma / 2)$ lies between any of these gaps and $y^{\prime \prime}$, and the length of $G_{1,1}$ is $\gamma$, we have that

$$
\operatorname{dist}\left(y^{\prime \prime}, G_{l, m}^{0}\right) \geq \frac{\gamma}{2}
$$

(2) The $2^{l-2}$ gaps remaining from step 1 are contained in an interval $I$ of length $(1-\gamma) / 2$. To this interval we apply the procedure in step 1 , splitting $I$ into two halves. Notice that there are exactly $2^{l-3}$ gaps of generation $l$ in each half. We denote the gaps on the half-interval that is farthest from $y^{\prime \prime}$ by $G_{l, m}^{1}$ (recall that $y^{\prime \prime}$ is not the center of the full interval $I$ ), with $m=1,2, \ldots, 2^{l-3}$. These gaps satisfy

$$
\operatorname{dist}\left(y^{\prime \prime}, G_{l, m}^{1}\right) \geq \frac{\gamma}{2} \frac{1-\gamma}{2}
$$

(3) Iterating this procedure, at each step $j$ we find exactly $2^{l-2-j}$ gaps of generation $l$, denoted by $G_{l, m}^{j}$ with $m=1,2, \ldots, 2^{l-2-j}$. They satisfy

$$
\operatorname{dist}\left(y^{\prime \prime}, G_{l, m}^{j}\right) \geq \frac{\gamma}{2}\left(\frac{1-\gamma}{2}\right)^{j}
$$

(4) We continue the iteration until $j=l-2$, which starts with only two gaps of generation $l$ left. The farthest from $y^{\prime \prime}$, denoted by $G_{l, 1}^{l-2}$, satisfies (6.21) with $j=l-2$. On the other hand, we denote the gap closest to $y^{\prime \prime}$ by $G_{l, 1}$.

Summarizing, among the $2^{l-1}$ gaps of generation $l$ we have selected one, called $G_{l, 1}$, in step 4. The gap $G_{l, 1}$ is the closest to $y^{\prime \prime}$ among those in generation $l$. The remaining $2^{l-1}-1$ gaps have been clustered into $l-1$ families $\left\{G_{l, m}^{0}\right\}_{m}, \ldots,\left\{G_{l, m}^{j}\right\}_{m}, \ldots,\left\{G_{l, m}^{l-2}\right\}_{m}$, where the $j$-th family contains $2^{l-2-j}$ gaps of generation $l$ which, in addition, satisfy 6.21. 
With this classification, we have

$$
\begin{aligned}
& \sum_{l=1}^{\infty} \sum_{m=1}^{2^{l-1}} \int_{G_{l, m}} \int_{B_{1 / 4}^{(n-1)}} \operatorname{dist}\left(x, \mathcal{C}_{n, \lambda}\right)^{-\alpha p-p}|x-y|^{\lambda-n} d x^{\prime} d x^{\prime \prime} \\
& \quad=\sum_{l=1}^{\infty} \int_{G_{l, 1}} \int_{B_{1 / 4}^{(n-1)}} \operatorname{dist}\left(x, \mathcal{C}_{n, \lambda}\right)^{-\alpha p-p}|x-y|^{\lambda-n} d x^{\prime} d x^{\prime \prime} \\
& \quad+\sum_{l=2}^{\infty} \sum_{j=0}^{l-2} \sum_{m=1}^{2^{l-2-j}} \int_{G_{l, m}^{j}} \int_{B_{1 / 4}^{(n-1)}} \operatorname{dist}\left(x, \mathcal{C}_{n, \lambda}\right)^{-\alpha p-p}|x-y|^{\lambda-n} d x^{\prime} d x^{\prime \prime} .
\end{aligned}
$$

There are two cases to study in the sequel since integrals over $G_{l, 1}$ and $G_{l, m}^{j}$ are qualitatively different. The key difference is that (6.21) allows us to control $|x-y|$ from below and the integrals over $G_{l, m}^{j}$ become independent of $y$. Thus, we can apply Lemma 6.5 to them. This is not possible for integrals over the gaps $G_{l, 1}$.

For the integrals over a gap $G_{l, 1}$, if $y^{\prime \prime} \in \bar{G}_{l, 1}$ then we can apply Lemma 6.4 directly. Instead, if $y^{\prime \prime} \notin \bar{G}_{l, 1}$, we move $y^{\prime \prime}$ to the closest boundary point of $G_{l, 1}$ from $y^{\prime \prime}$, and with this procedure the integral becomes larger (since all the distances from points in $B_{1 / 4}^{(n-1)} \times G_{l, 1}$ decrease). With this new point $y^{\prime \prime}$ the integral can be bounded using Lemma 6.4.

In fact, when we assume $y^{\prime \prime} \in \overline{G_{l, 1}}$ Lemma 6.4 yields

$$
\begin{aligned}
& \sum_{l=1}^{\infty} \int_{G_{l, 1}} \int_{B_{1 / 4}^{(n-1)}} \operatorname{dist}\left(x, \mathcal{C}_{n, \lambda}\right)^{-\alpha p-p}|x-y|^{\lambda-n} d x^{\prime} d x^{\prime \prime} \\
& \leq C \sum_{l=1}^{\infty}\left(\left(\frac{1-\gamma}{2}\right)^{(\lambda-\alpha p-p)(l-1)}+l\left(\frac{1-\gamma}{2}\right)^{l-1}\right) \leq C,
\end{aligned}
$$

uniformly in $y$, since $(1-\gamma) / 2<1$ and $\lambda-\alpha p-p>0$ by hypothesis (6.6).

On the other hand, given a gap $G_{l, m}^{j}$, by (6.21) we have

$$
\begin{aligned}
& \int_{G_{l, m}^{j}} \int_{B_{1 / 4}^{(n-1)}} \operatorname{dist}\left(x, \mathcal{C}_{n, \lambda}\right)^{-\alpha p-p}|x-y|^{\lambda-n} d x^{\prime} d x^{\prime \prime} \\
& \leq C\left(\frac{1-\gamma}{2}\right)^{(\lambda-n) j} \int_{G_{l, m}^{j}} \int_{B_{1 / 4}^{(n-1)}} \operatorname{dist}\left(x, \mathcal{C}_{n, \lambda}\right)^{-\alpha p-p} d x^{\prime} d x^{\prime \prime}
\end{aligned}
$$

Then, Lemma 6.5 leads to

$$
\begin{aligned}
& \int_{G_{l, m}^{j}} \int_{B_{1 / 4}^{(n-1)}} \operatorname{dist}\left(x, \mathcal{C}_{n, \lambda}\right)^{-\alpha p-p}|x-y|^{\lambda-n} d x^{\prime} d x^{\prime \prime} \\
& \leq C\left(\left(\frac{1-\gamma}{2}\right)^{(\lambda-n) j+(n-\alpha p-p)(l-1)}+l\left(\frac{1-\gamma}{2}\right)^{(\lambda-n) j+l-1}\right)
\end{aligned}
$$


uniformly in $m$. Observe that by our choice of $\gamma$, we have $\left(\frac{1-\gamma}{2}\right)^{\lambda-n}=2$ and thus

$$
\begin{aligned}
& \sum_{l=2}^{\infty} \sum_{j=0}^{l-2} \sum_{m=1}^{2^{l-2-j}} \int_{G_{l, m}^{j}} \int_{B_{1 / 4}^{(n-1)}} \operatorname{dist}\left(x, \mathcal{C}_{n, \lambda}\right)^{-\alpha p-p}|x-y|^{\lambda-n} d x^{\prime} d x^{\prime \prime} \\
& \leq C \sum_{l=2}^{\infty} \sum_{j=0}^{l-2} 2^{l-2-j}\left(\left(\frac{1-\gamma}{2}\right)^{(\lambda-n) j+(n-\alpha p-p)(l-1)}+l\left(\frac{1-\gamma}{2}\right)^{(\lambda-n) j+l-1}\right) \\
& =\frac{C}{2} \sum_{l=2}^{\infty} \sum_{j=0}^{l-2}\left(\left(\frac{1-\gamma}{2}\right)^{(\lambda-\alpha p-p)(l-1)}+l\left(\frac{1-\gamma}{2}\right)^{(\lambda-n+1)(l-1)}\right) \\
& =\frac{C}{2} \sum_{l=2}^{\infty}\left((l-1)\left(\frac{1-\gamma}{2}\right)^{(\lambda-\alpha p-p)(l-1)}+l(l-1)\left(\frac{1-\gamma}{2}\right)^{(\lambda-n+1)(l-1)}\right) \leq C,
\end{aligned}
$$

uniformly in $y$, since $(1-\gamma) / 2<1, \lambda-\alpha p-p>0$, and $\lambda>n-1$ by hypothesis.

Then, (6.20), (6.22), (6.23), and (6.24) give

$$
\int_{-\frac{1}{2}}^{\frac{1}{2}} \int_{B_{1 / 4}^{(n-1)}} \operatorname{dist}\left(x, \mathcal{C}_{n, \lambda}\right)^{-\alpha p-p}|x-y|^{\lambda-n} d x^{\prime} d x^{\prime \prime} \leq C
$$

uniformly in $y$, and the proof is complete.

We conclude the article with the proof of Lemma 6.6.

Proof of Lemma 6.6. Note first that the support of $u_{\alpha, n}$, given by (6.7), is included in $\bar{B}_{1 / 4}^{(n-1)} \times[-1,1]$. Hence, for any given $y \in \bar{B}_{1}^{(n)}$ we have

$$
\int_{B_{1}^{(n)}}\left|\nabla u_{\alpha, n}(x)\right|^{p}|x-y|^{\lambda-n} d x \leq C \int_{-1}^{1} \int_{B_{1 / 4}^{(n-1)}} \operatorname{dist}\left(x, \mathcal{C}_{n, \lambda}\right)^{-\alpha p-p}|x-y|^{\lambda-n} d x^{\prime} d x^{\prime \prime},
$$

where we have used that the modulus of the gradient of a distance function is equal to 1 a.e. Then, our goal is to show that

$$
\begin{aligned}
& \sup _{y \in \bar{B}_{1}^{(n)}} \int_{-1}^{1} \int_{B_{1 / 4}^{(n-1)}} \operatorname{dist}\left(x, \mathcal{C}_{n, \lambda}\right)^{-\alpha p-p}|x-y|^{\lambda-n} d x^{\prime} d x^{\prime \prime} \\
& \leq C \sup _{y^{\prime}=0,\left|y^{\prime \prime}\right| \leq \frac{1}{2}} \int_{-\frac{1}{2}}^{\frac{1}{2}} \int_{B_{1 / 4}^{(n-1)}} \operatorname{dist}\left(x, \mathcal{C}_{n, \lambda}\right)^{-\alpha p-p}|x-y|^{\lambda-n} d x^{\prime} d x^{\prime \prime},
\end{aligned}
$$

from which (6.19) follows.

First we will prove that the supremum over $y \in \bar{B}_{1}^{(n)}$ is bounded by the supremum over the axis, i.e., $y \in\{0\} \times[-1,1]$. Then, that it actually suffices that $\left|y^{\prime \prime}\right| \leq 1 / 2$ instead of $\left|y^{\prime \prime}\right| \leq 1$, and finally that it is enough to integrate $x^{\prime \prime}$ over $[-1 / 2,1 / 2]$ instead of the whole $[-1,1]$. In doing these we will use twice the monotonicity result in Lemma 2.1. 


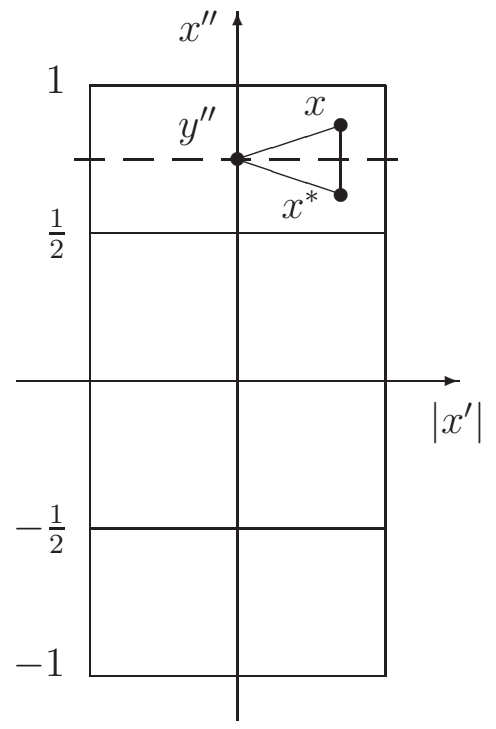

Figure 4. Monotonicity argument for $J_{2}$ in the proof of Lemma 6.6.

Therefore, consider $y \in \bar{B}_{1}^{(n)}$, and let us show that

$$
J_{1}(y):=\int_{-1}^{1} \int_{B_{1 / 4}^{(n-1)}} \operatorname{dist}\left(x, \mathcal{C}_{n, \lambda}\right)^{-\alpha p-p}|x-y|^{\lambda-n} d x^{\prime} d x^{\prime \prime} \leq J_{1}\left(0, y^{\prime \prime}\right),
$$

where $y=\left(y^{\prime}, y^{\prime \prime}\right) \in \bar{B}_{1}^{(n)}$, and thus $y^{\prime \prime} \in[-1,1]$. In fact, upon a rotation in the $x^{\prime}$ variables, we can assume that $y^{\prime}=\left(y_{1}, 0\right) \in \mathbb{R} \times \mathbb{R}^{n-2}$ with $y_{1} \geq 0$. Hence, we are under the hypotheses of Lemma 2.1 with $\Omega=B_{1 / 4}^{(n-1)} \times[-1,1], h(z)=\operatorname{dist}\left(z, \mathcal{C}_{n, \lambda}\right)^{-\alpha p-p}$ (which is non-increasing with respect to $z_{1}$ in $\left.\left\{z_{1} \geq 0\right\}\right), \theta=n-\lambda$, and $\eta=0$. Therefore, Lemma 2.1 gives that $J_{1}$ is non-increasing with respect to $y_{1}$ in $[0, \infty)$. Hence we conclude that $J_{1}(y) \leq J_{1}\left(0, y^{\prime \prime}\right)$, as desired.

A similar argument shows that we just need to consider the case $\left|y^{\prime \prime}\right| \leq 1 / 2$ instead of $\left|y^{\prime \prime}\right| \leq 1$. In fact, define

$$
J_{2}\left(y^{\prime \prime}\right):=\int_{-1}^{1} \int_{B_{1 / 4}^{(n-1)}} \operatorname{dist}\left(x, \mathcal{C}_{n, \lambda}\right)^{-\alpha p-p}\left(\left|x^{\prime}\right|^{2}+\left(x^{\prime \prime}-y^{\prime \prime}\right)^{2}\right)^{\frac{\lambda-n}{2}} d x^{\prime} d x^{\prime \prime}
$$

and assume $\left|y^{\prime \prime}\right| \geq 1 / 2$. By symmetry, we can assume $y^{\prime \prime} \geq 1 / 2$. In order to apply Lemma 2.1, we take $x^{\prime \prime}$ as the direction $e_{1}$ which is "privileged" in the lemma, while the rest of the hypotheses are fulfilled for $\left(z^{\prime \prime}, z^{\prime}\right) \in \Omega=[-1,1] \times B_{1 / 4}^{(n-1)}, h\left(z^{\prime \prime}, z^{\prime}\right)=$ $\operatorname{dist}\left(\left(z^{\prime}, z^{\prime \prime}\right), \mathcal{C}_{n, \lambda}\right)^{-\alpha p-p}$ (which is non-increasing with respect to $z^{\prime \prime}$ in $\left\{z^{\prime \prime} \geq 1 / 2\right\}$ ), $\theta=n-\lambda$, and $\eta=1 / 2$, since the set $\mathcal{C}_{n, \lambda}$ is contained in $\{0\} \times[-1 / 2,1 / 2]$ (see Figure 4). Then, Lemma 2.1 gives that $J_{2}\left(y^{\prime \prime}\right)$ is non-increasing with respect to $y^{\prime \prime}$ in $[1 / 2, \infty)$, and therefore it is enough to study the case $y^{\prime \prime} \leq 1 / 2$. The case $y^{\prime \prime} \leq-1 / 2$ follows similarly by taking $\eta=-1 / 2$ in the lemma. 
Finally, let $y=\left(0, y^{\prime \prime}\right)$ with $\left|y^{\prime \prime}\right| \leq 1 / 2$. Notice that for every $x \in B_{1 / 4}^{(n-1)} \times[1 / 2,1]$ and its reflected $x^{*}$ with respect to $\left\{x^{\prime \prime}=1 / 2\right\}$, we have $\left|x^{*}-y\right| \leq|x-y|$ and $\operatorname{dist}\left(x^{*}, \mathcal{C}_{n, \lambda}\right) \leq \operatorname{dist}\left(x, \mathcal{C}_{n, \lambda}\right)$, which give

$$
\operatorname{dist}\left(x, \mathcal{C}_{n, \lambda}\right)^{-\alpha p-p}|x-y|^{\lambda-n} \leq \operatorname{dist}\left(x^{*}, \mathcal{C}_{n, \lambda}\right)^{-\alpha p-p}\left|x^{*}-y\right|^{\lambda-n} .
$$

This leads to

$$
\begin{aligned}
\int_{\frac{1}{2}}^{1} \int_{B_{1 / 4}^{(n-1)}} & \operatorname{dist}\left(x, \mathcal{C}_{n, \lambda}\right)^{-\alpha p-p}|x-y|^{\lambda-n} d x^{\prime} d x^{\prime \prime} \\
& \leq \int_{0}^{\frac{1}{2}} \int_{B_{1 / 4}^{(n-1)}} \operatorname{dist}\left(x, \mathcal{C}_{n, \lambda}\right)^{-\alpha p-p}|x-y|^{\lambda-n} d x^{\prime} d x^{\prime \prime},
\end{aligned}
$$

and similarly for the integral over $B_{1 / 4}^{(n-1)} \times\left[-1,-\frac{1}{2}\right]$. Therefore,

$$
\begin{aligned}
& \int_{-1}^{1} \int_{B_{1 / 4}^{(n-1)}} \operatorname{dist}\left(x, \mathcal{C}_{n, \lambda}\right)^{-\alpha p-p}\left(\left|x^{\prime}\right|^{2}+\left(x^{\prime \prime}-y^{\prime \prime}\right)^{2}\right)^{\frac{\lambda-n}{2}} d x^{\prime} d x^{\prime \prime} \\
& \leq 2 \int_{-\frac{1}{2}}^{\frac{1}{2}} \int_{B_{1 / 4}^{(n-1)}} \operatorname{dist}\left(x, \mathcal{C}_{n, \lambda}\right)^{-\alpha p-p}\left(\left|x^{\prime}\right|^{2}+\left(x^{\prime \prime}-y^{\prime \prime}\right)^{2}\right)^{\frac{\lambda-n}{2}} d x^{\prime} d x^{\prime \prime}
\end{aligned}
$$

which completes the proof of the lemma.

Acknowledgements: The first author would like to thank Joan Orobitg and Joan Verdera for a stimulating discussion on the topic of this paper. The authors also thank Giuseppe Mingione for interesting comments and for bringing [2, 6, 10] to their attention after the completion of this manuscript, as well as the referee for some appropriate remarks and references [11, 13].

\section{REFERENCES}

[1] Adams, D. R.; A note on Riesz potentials, Duke Math. J. 42 (1975), 765-778.

[2] Adams, D. R., Lewis, J. L.; On Morrey-Besov inequalities, Studia Math. 74 (1982), 169-182.

[3] Adams, D. R., Xiao, J.; Morrey potentials and harmonic maps, Comm. Math. Phy. 308 (2011), 439-456.

[4] Adams, D. R., Xiao, J.; Erratum to: Morrey potentials and harmonic maps, Comm. Math. Phys. 339 (2015), 769-771.

[5] Adams, D. R., Xiao, J.; Restrictions of Riesz-Morrey potentials, Ark. Mat. 54 (2016), 201-231.

[6] Bensoussan, A., Frehse, J.; Regularity results for nonlinear elliptic systems and applications, Applied Mathematical Sciences, vol. 151, Berlin: Springer, 2002.

[7] Cabré, X., Figalli, A., Ros-Oton, X., Serra J.; Stable solutions to semilinear elliptic equations are smooth up to dimension 9, preprint arXiv:1907.09403.

[8] Cabré, X., Ros-Oton, X.; Sobolev and isoperimetric inequalities with monomial weights, J. Differential Equations 255 (2013), 4312-4336.

[9] Falconer, K.; Fractal geometry: mathematical foundations and applications, John Wiley \& Sons, 2004.

[10] Fonseca, I., Malý, J., Mingione, G.; Scalar Minimizers with Fractal Singular Sets, Arch. Rational Mech. Anal. 172 (2004), 295-307.

[11] Hou, S., Xiao, J.; Cordes-Nirenberg's imbedding and restricting with application to an elliptic equation, Commun. Contemp. Math. 20 (2018), no. 7, 1750080, 20 pp. 
[12] Schoen, R., Yau, S.-T.; Conformally flat manifolds, Kleinian groups and scalar curvature, Invent. Math. 92 (1988), 47-71.

[13] Talenti, G.; Best constant in Sobolev inequality, Ann. Mat. Pura Appl. 110 (1976), 353-372.

[14] Talenti, G.; A weighted version of a rearrangement inequality, Ann. Univ. Ferrara 43 (1997), 121-133.

X.C. ${ }^{1,2,3}$ — ${ }^{1}$ ICREA, PG. Lluis Companys 23, 08010 Barcelona, Spain \& ${ }^{2}$ Universitat Politècnica de Catalunya, Departament de Matemàtiques, Diagonal 647, 08028 Barcelona, Spain \& ${ }^{3}$ BGSmath, Campus de Bellaterra, Edifici C, 08193 Bellaterra, Spain.

Email address: xavier.cabre@upc.edu

F.C. - Department of Mathematics, Wayne State University, 656 W. Kirby, DeTROIT, MI 48202, USA.

Email address: fcharro@wayne.edu 